TRANSACTIONS OF THE

AMERICAN MATHEMATICAL SOCIETY

Volume 350, Number 2, February 1998, Pages 571-613

S $0002-9947(98) 01773-5$

\title{
HYPERBOLIC GROUPS AND FREE CONSTRUCTIONS
}

\author{
O. KHARLAMPOVICH AND A. MYASNIKOV
}

\begin{abstract}
It is proved that the property of a group to be hyperbolic is preserved under HHN-extensions and amalgamated free products provided the associated (amalgamated) subgroups satisfy certain conditions. Some more general results about the preservation of hyperbolicity under graph products are also obtained. Using these results we describe the $\mathbf{Q}$-completion ( $\mathbf{Q}$ is the field of rationals) $G^{\mathbf{Q}}$ of a torsion-free hyperbolic group $G$ as a union of an effective chain of hyperbolic subgroups, and solve the conjugacy problem in $G^{\mathrm{Q}}$.
\end{abstract}

Hyperbolic groups have been the subject of intensive investigation since the work of Gromov [8]. Let $G=\langle J, \mathcal{R}\rangle$ be a finitely presented group with a set of generators $J$ and a set of relators $\mathcal{R}$. A word $W$ in the alphabet $J^{ \pm 1}$ is equal to 1 in $G$ if and only if there is an equality

$$
W=\prod_{i=1}^{n} S_{i}^{-1} R_{i}^{ \pm 1} S_{i}
$$

in the free group $F=F(J)$, where $S_{i} \in F$ and $R_{i} \in \mathcal{R}$. The group $G$ is hyperbolic if there exists a linear function bounding the minimal number of factors $n=n(W)$ in (1) depending on the length $\|W\|$ of the word $W$. This definition does not depend on the choice of the presentation of $G$.

In his book ([8], §3.3) M.Gromov claimed that if $G_{1}$ and $G_{2}$ are torsion-free hyperbolic and $U$ and $V$ are maximal cyclic subgroups in $G_{1}$ and $G_{2}$ respectively, then the amalgamated free product $G_{1} *_{U=V} G_{2}$ is also hyperbolic.

On the other hand the group $\langle x\rangle *_{x^{n}=y^{m}}\langle y\rangle$ for $|n|,|m|>1$ is not hyperbolic, because it contains a free abelian subgroup with generators $x y$ and $x^{n}$.

The Baumslag-Solitar groups $\operatorname{BS}(m, n)=\left\langle x, t \mid t^{-1} x^{m} t=x^{n}\right\rangle$ provide examples of HNN-extensions of hyperbolic group (with cyclic associated subgroups) that are not hyperbolic.

In [4] it has been shown that an amalgamated product of two hyperbolic groups with a cyclic subgroup amalgamated is automatic and that an amalgamated product of two finitely generated free groups with a finitely generated subgroup amalgamated is acynchronously automatic. It has been also proved in [4] that if $G$ is an HNN-extension of a finitely generated free group with finitely many stable letters and if the associated subgroups are all finitely generated, then $G$ is asynchronously automatic. Under the additional assumption of "speed-matching", such HNN-extensions are shown to be automatic in [16]. (Notice that the class of automatic groups is contained in the class of asynchronously automatic groups and

Received by the editors July 7, 1994 and, in revised form, January 18, 1996.

1991 Mathematics Subject Classification. Primary 20F06, 20 E06.

The first author was supported by grants from NSERC and FCAR.

(C) 1998 American Mathematical Society 
contains the class of hyperbolic groups.) In [3], [6] it was proved that if an amalgamated free product $G_{1} *_{U} G_{2}$ is automatic then both groups $G_{1}$ and $G_{2}$ are automatic provided the amalgamated subgroup $U$ is finite.

In [5] Bestvina and Feighn proved the combination theorem for negatively curved spaces and as a corollary obtained the result which we also formulate in this paper as Corollary 2.

We define a subgroup $U$ of a group $G$ to be conjugate separated if the set $\{u \in$ $\left.U \mid u^{x} \in U\right\}$ is finite for all $x \in G \backslash U$.

Let us introduce the construction of a separated HNN-extension of a group $G$.

Definition 1. Suppose that $U$ and $V$ are subgroups of $G, \psi: U \rightarrow V$ is an isomorphism, either $U$ or $V$ is conjugate separated, and the set $U \cap g^{-1} V g$ is finite for all $g \in G$. Then the HNN-extension $\left\langle G, t \mid t^{-1} u t=u^{\psi}, u \in U\right\rangle$ is called separated.

Let $a, b \in G$; by $|a-b|_{G}$ we denote the distance between the points $a$ and $b$ in the Cayley graph of $G$ (see the definition below). If it is clear from the context in which group the distance is taken, we will just write $|a-b|$.

A finitely generated subgroup $U$ of a hyperbolic group $G$ is said to be quasiisometrically embedded if there is a constant $\lambda=\lambda(U)$ such that $|1-a|_{U} \leq \lambda|1-a|_{G}$ for any element $a \in U$. Every quasiisometrically embedded subgroup of a hyperbolic group is itself hyperbolic.

It is not hard to see that a subgroup of a hyperbolic group is quasiisometrically embedded if and only if it is quasiconvex (the definition of quasiconvexity can be found in Section 6).

Theorem 1. If $\mathcal{G}$ is a hyperbolic group and $\mathcal{H}=\left\langle\mathcal{G}, t \mid \mathcal{U}^{t}=\mathcal{V}\right\rangle$ is a separated $H N N$-extension such that the subgroups $\mathcal{U}$ and $\mathcal{V}$ are quasiisometrically embedded in $\mathcal{G}$, then $\mathcal{H}$ is hyperbolic.

Theorem 2. Let $\mathcal{G}_{1}, \mathcal{G}_{2}$ be hyperbolic groups, $\mathcal{U} \leq \mathcal{G}_{1}, \mathcal{V} \leq \mathcal{G}_{2}$ quasiisometrically embedded, and $\mathcal{U}$ conjugate separated in $\mathcal{G}_{1}$. Then the group $\mathcal{G}_{1} * \mathcal{U}=\mathcal{\nu} \mathcal{G}_{2}$ is hyperbolic.

As corollaries we have the following results.

Corollary 1. If $\mathcal{G}$ is a hyperbolic group, $\mathcal{A}$ and $\mathcal{B}$ isomorphic virtually cyclic subgroups, then the $H N N$-extension $\mathcal{H}=\left\langle\mathcal{G}, t \mid \mathcal{A}^{t}=\mathcal{B}\right\rangle$ is hyperbolic if and only if it is separated.

Corollary 2 ([5]). Let $\mathcal{G}_{1}, \mathcal{G}_{2}$ be hyperbolic groups, $\mathcal{A} \leq \mathcal{G}_{1}, \mathcal{B} \leq \mathcal{G}_{2}$, virtually cyclic. Then the group $\mathcal{G}_{1} *_{\mathcal{A}=\mathcal{B}} \mathcal{G}_{2}$ is hyperbolic if and only if either $\mathcal{A}$ is conjugate separated in $\mathcal{G}_{1}$ or $\mathcal{B}$ is conjugate separated in $\mathcal{G}_{2}$.

The assertion of our Corollary 1 contradicts the assertion of the last corollary (HNNs over virtually cyclics) in [5] (there is an omission in this corollary in [5]). The group given by the presentation $K=\left\langle a, b, t \mid t^{-1} a^{2} t=b^{2}\right\rangle$ is a counterexample. The group is obviously not hyperbolic, but it satisfies condition (2) in the last corollary in [5].

After our paper was ready, A.Yu. Olshanskii informed us that he and his student K. Mikhajlovskii [11] independently obtained the results formulated in Corollaries 1 and 2. Corollary 2 is also proved in [7].

Corollary 3. HNN-extensions (amalgamated products) of hyperbolic groups with finite associated (amalgamated) subgroups are hyperbolic. 
Corollary 4. Separated HNN-extensions of a free group with finitely generated associated subgroups are hyperbolic.

The condition in Theorems 1 and 2 can be weakened (see Section 5). The proof of our results uses a geometric interpretation by Van Kampen diagrams of the deducibility of relations in a group from the defining relations, as well as the hyperbolicity of the Cayley graph $\Gamma(\mathcal{G})$ of a hyperbolic group $\mathcal{G}$.

In Section 6 we prove some results on quasiconvexity (Theorems 4, 5, 6 and 7).

In Section 7 we apply our results to exponential groups.

Let $A$ be an arbitrary associative ring with identity and $G$ a group. Fix an action of the ring $A$ on $G$, i.e. a map $G \times A \rightarrow G$. The result of the action of $\alpha \in A$ on $g \in G$ is written as $g^{\alpha}$. Groups with $A$-actions satisfying axioms 1)-4) in Section 7 are called $A$-groups. In particular, an arbitrary group $G$ is a $\mathbf{Z}$-group. In the case where $A$ is the field of rationals $\mathbf{Q}, \mathbf{Q}$-completions of groups (see definition in Section 7) were studied by G. Baumslag in [2], [1]. A-completions for arbitrary rings $A$ were investigated in [12].

In [1] it was proved that for a free group $F$ the word problem in the $\mathbf{Q}$-completion $F^{\mathbf{Q}}$ is solvable. The proof was based on the residual finiteness of some specific subgroups of $F^{\mathbf{Q}}$, so the problem was posed of finding a "direct" proof of the solvability of the word problem using normal forms of elements in $F^{\mathbf{Q}}$. Moreover, in the same article Baumslag mentioned that the conjugacy problem in $F^{\mathbf{Q}}$ is still open.

In Section 7 we describe the $\mathbf{Q}$-completion $G^{\mathbf{Q}}$ of a torsion-free hyperbolic group $G$ as the union of an effective chain of hyperbolic subgroups. This allows one to apply techniques of hyperbolic group theory to solve various algorithmic problems in $G^{\mathbf{Q}}$, in particular, to solve the conjugacy problem (Theorem 10) and to construct effectively some natural normal forms for its elements, induced by the normal forms of amalgamated free products (Theorem 9). A free group $F$ is hyperbolic, so one can answer two of Baumslag's questions [1] mentioned above.

\section{QUASIGEODESIC POLYGONS IN HYPERBOLIC GROUPS}

Let us recall some notions from the theory of hyperbolic spaces.

Let $X$ be a metric space, $|x-y|$ the distance between points $x, y \in X$. If one fixes a point $o \in X$ then Gromov's product $(x \cdot y)_{o}$ is by definition

$$
(x \cdot y)_{o}=1 / 2(|x-o|+|y-o|-|x-y|) .
$$

The space $X$ is called $\delta$-hyperbolic (for some fixed constant $\delta \geq 0$ ) if for all points $x, y, z, o \in X$

$$
(x \cdot y)_{o} \geq \min \left((x \cdot z)_{o},(y \cdot z)_{o}\right)-\delta .
$$

And $X$ is called hyperbolic if it is $\delta$-hyperbolic for some $\delta \geq 0$

A geodesic segment between points $x, y \in X$ is an isometric map $[0,|x-y|] \longrightarrow X$ sending 0 to $x$ and $|x-y|$ to $y$. Its image will also be called a geodesic segment; $[x, y]$ is the notation for some fixed geodesic segment between $x$ and $y$. A metric space is called geodesic if every pair of points can be connected by a geodesic segment. We shall call a geodesic $\delta$-hyperbolic space simply a $\delta$-space.

An example of a geodesic space is the realization of the Cayley graph $\Gamma(G)=$ $\Gamma(G, J)$ of a group $G$ with a fixed generating system $J$. Recall that the vertices of $\Gamma(G)$ are elements of $G$, and the elements $g, h=g a, a \in J$, are connected by an edge $e=(g, a)$ having label $\phi(e)=a \in J$. The label of a path is the product of the 
labels of the edges of this path. Let us endow each edge $e$ with the metric of the unit segment $[0,1]$. By definition now the distance $|x-y|$ between points $x$ and $y$ is the length of a shortest path in $\Gamma(G)$ between $x$ and $y$.

A finitely presented group $G$ is hyperbolic if and only if $\Gamma(G)$ is a hyperbolic space [8]. If $g, h \in G$ then by definition $(g \cdot h)=(g \cdot h)_{1}$ is the Gromov product in $\Gamma(G)$, where 1 is the identity of $G$. Let $p$ be a path and $x$ a point. Let $|x, p|$ denote the distance between the point $x$ and the path $p$ and $|x,[y, z]|$ denote the distance between the point $x$ and the geodesic segment $[y, z]$.

Lemma 1 ([14], Lemma 1.5). For each geodesic triangle $\left[x_{1}, x_{2}, x_{3}\right]$ in a $\delta$-space, there are points $y_{i} \in\left[x_{i-1}, x_{i+1}\right]$ (indices are considered modulo 3) such that

for any point $u \in\left[x_{i}, y_{i \pm 1}\right]$.

$$
\begin{gathered}
\left|x_{i}-y_{i-1}\right|=\left|x_{i}-y_{i+1}\right|=\left(x_{i-1} \cdot x_{i+1}\right)_{x_{i}} \\
\left|y_{i}-y_{i-1}\right| \leq 4 \delta \text { and }\left|u,\left[x_{i}, y_{i \pm 1}\right]\right| \leq 4 \delta
\end{gathered}
$$

It is easy to verify that for a hyperbolic group $G$ we have in $\Gamma(G)$

$$
(x \cdot z)_{y}=(\phi([y, x]) \cdot \phi([y, z])) .
$$

We can rewrite the equalities from the lemma in the following form:

$$
\left|x_{i}-y_{i-1}\right|=\left|x_{i}-y_{i+1}\right|=\left(\phi\left[x_{i}, x_{i-1}\right] \cdot \phi\left[x_{i}, x_{i+1}\right]\right) .
$$

A path $p$ with the natural parametrisation by length in $\Gamma(G)$ is called $(\lambda, \mu)$ quasigeodesic for some $\lambda \geq 0$ and $\mu \geq 0$, if for any points $p(s)$ and $p(t)$

$$
\lambda|s-t|-\mu \leq|p(s)-p(t)| .
$$

Denote by $q_{-}$(resp. $q_{+}$) the initial (resp. terminal) vertex of a path $q$. A word in the generators of $G$ is called geodesic (resp. quasigeodesic) if the corresponding path is geodesic (resp. quasigeodesic) in the Cayley graph of $G$.

If a subgroup $U$ is quasiisometrically embedded in a hyperbolic group $G$ then every geodesic in $U$ is a $(\lambda, 0)$-quasigeodesic in $G$.

Lemma 2 ([14], Lemma 1.9). There exists a constant $H_{1}=H_{1}(\delta, \lambda, \mu)$ such that for any $(\lambda, \mu)$-quasigeodesic path $p$ in a $\delta$-space and any geodesic path $q$ with the conditions $p_{-}=q_{-}$and $p_{+}=q_{+}$, the inequalities $|u, p|<H_{1}$ and $|v, q|<H_{1}$ hold for any points $u \in q$ and $v \in p$.

As in [14], call two paths $p$ and $q C$-bound if $\left(\left|p_{-}-q_{-}\right|,\left|p_{+}-q_{+}\right|\right) \leq C$.

Lemma 3 ([14], Lemma 1.7). Let $\left[x_{1}, \ldots, x_{4}\right]$ be a geodesic quadrangle in a $\delta$ space and

$$
\left|x_{1}-x_{2}\right|>4 \max \left(\left|x_{1}-x_{4}\right|,\left|x_{2}-x_{3}\right|\right) \text {. }
$$

Then, there exist $8 \delta$-bound subsegments $p$ and $q$ of the segments $\left[x_{1}, x_{2}\right]$ and $\left[x_{3}, x_{4}\right]$ such that

$$
\min (|p|,|q|) \geq(7 / 20)\left|x_{1}-x_{2}\right|-8 \delta .
$$

Now we fix some notation and introduce constants $\delta, \lambda, c, H_{1}$, that will be used below without reference. Let $\mathcal{G}$ be the hyperbolic group from Theorem $1, J$ a fixed set of generators of $\mathcal{G}$. Suppose $G$ is $\delta$-hyperbolic. Let $\mathcal{U}$ and $\mathcal{V}$ be the subgroups of $\mathcal{G}$ from Theorem 1, quasiisometrically embedded with the constant $\lambda$. Let $\mathcal{U}$ be conjugate separated.

We introduce two length functions on the group $\mathcal{G}$. If $g \in \mathcal{G}$ then $|g|=|g-1|$ in $\Gamma(\mathcal{G}, J)$. In other words, $|g|$ is the length of a shortest word in the alphabet $J$ 
representing $g$ in $\mathcal{G}$. We also consider words in the alphabet $J$ to be elements of $\mathcal{G}$. The notation $W \equiv V$ means the equality of words, and $W=V$ means the equality of elements. Suppose $V$ is a word in the alphabet $J$; we say that $W$ is a geodesic word such that $W=V$ if $W$ is a shortest word representing in $\mathcal{G}$ the same element as $V$.

By $\|W\|=\|W\|_{\mathcal{G}}$ we denote the length of the word $W$. Clearly the length $\|W\|$ can be greater then $|W|$.

We now fix some presentation of $\mathcal{H}$. Let $A=\left\{a_{1}, \ldots, a_{n}\right\}$ be a generating set of $\mathcal{U}, \psi: \mathcal{U} \rightarrow \mathcal{V}$ an isomorphism, $b_{i}=\psi\left(a_{i}\right), i=1, \ldots, n$, and $B=\left\{b_{1}, \ldots, b_{n}\right\}$. Then $a_{i}^{t}=b_{i}$ is a defining relation in $\mathcal{H}$. Let $c=\max \left\{\left|a_{1}\right|, \ldots\left|a_{n}\right|,\left|b_{1}\right|, \ldots\left|b_{n}\right|\right\}$.

One can consider two metrics on $\mathcal{U}$, one induced from $\mathcal{G}$ and the other the word metric in the generators from $A$ (for $W \in \mathcal{U}$ we denote the latter by $|W|_{\mathcal{U}}$ ). We also denote the length of the word $W \in \mathcal{U}$ in the generators $a_{1}, \ldots, a_{n}$ by $\|W\|_{\mathcal{U}}$. Then $|W|_{\mathcal{U}} \leq\|W\|_{\mathcal{U}} \leq\|W\| \leq c\|W\|_{\mathcal{U}}$. It is also clear that $|W|_{\mathcal{U}} \leq$ $\lambda|W|,|W|_{\mathcal{V}} \leq \lambda|W|$ and $|W| \leq c|W|_{\mathcal{U}}$. For $V \in \mathcal{V}$ we have $|V| \leq c|V|_{\mathcal{V}}$. If $U \in \mathcal{U}$ and $U\left(a_{1}(J), \ldots, a_{n}(J)\right)$ is a geodesic word in $\mathcal{U}$ in the generators in $A$, then we say that $U$ is a $\mathcal{U}$-geodesic word (or a geodesic in $\mathcal{U}$ ). The same for $\mathcal{V}$.

If we have a path $p$ in the Cayley graph of $\mathcal{G}$ such that $\phi(p)$ is a word in the generators in $A$ (resp. $B$ ) which are words in the generators of $G$, then the vertices of $p$ corresponding to the beginnings and ends of the generators in $A$ (resp. $B$ ) will be called phase vertices (phase points). Phase vertices depend on the way we write $\phi(p)$ as a word in the generators of $\mathcal{U}$ (respectively $\mathcal{V}$ ). We call a word $W$ cyclically minimal if it has minimal length among all words conjugated to $W$ in $\mathcal{G}$.

Let $H_{1}$ be the constant obtained for the group $\mathcal{G}$ as in Lemma 2.

Lemma 4. Let $H_{2}=H_{1}+c, H=2 H_{2}+8 \delta$. There is a constant $M_{0}=M_{0}(\mathcal{G}, \mathcal{U}, \mathcal{V})$ such that for any $\mathcal{U}$-geodesic words $u, \bar{u}, \mathcal{V}$-geodesic words $v, \bar{v}$, and geodesic words $X, Y \in \mathcal{G}$,

1) the equality $X u Y \bar{u}=1$ for $|u| \geq M_{0}$ implies

$$
4 \cdot \max (|X|,|Y|) \geq|u|,|\bar{u}|,
$$

or $X$ and $Y$ belong to $\mathcal{U}$.

2) the equality $X u Y v=1$ implies $\max (|u|,|v|)<M_{0}$ or $4 \cdot \max (|X|,|Y|) \geq$ $|u|,|v|$,

3) the equality $X v Y \bar{v}=1$ for $|v| \geq M_{0}$ implies one of the following:

1. $4 \cdot \max (|X|,|Y|) \geq|v|,|\bar{v}|$,

2. $X$ and $Y$ belong to $\mathcal{V}$,

3. there are elements $T_{1}$ and $T_{2}$ such that $\left|T_{i}\right|<H, v \equiv v_{1} v_{3} v_{2}, \bar{v} \equiv \bar{v}_{2} \bar{v}_{3} \bar{v}_{1}$,

$$
\begin{gathered}
X v_{1} T_{1}^{-1} \bar{v}_{1}=1, T_{1} v_{3} T_{2}^{-1} \bar{v}_{3}=1, T_{2} v_{2} Y \bar{v}_{2}=1 \\
\text { and }\left|v_{1}\right|,\left|\bar{v}_{1}\right| \leq 4 \max \{|X|, H\},\left|v_{2}\right|,\left|\bar{v}_{2}\right| \leq 4 \max \{|Y|, H\} .
\end{gathered}
$$

Proof. See Fig. 1. Let $W_{1}, W_{2} \in\{u, \bar{u}, v, \bar{v}\}$, and suppose we have an equality $X W_{1} Y W_{2}=1$. If $X=Y=1$ there is nothing to prove. Suppose $X \neq 1$. By the condition of the lemma, there is a quadrangle $p^{1} q^{1} p^{2} q^{2}$ in the Cayley graph $\Gamma(\mathcal{G})$ such that $\phi\left(p^{1}\right)=X, \phi\left(q^{1}\right)=W_{1}, \phi\left(p^{2}\right)=Y, \phi\left(q^{2}\right)=W_{2}$, the paths $q^{1}, q^{2}$ are quasigeodesic and $p^{1}, p^{2}$ are geodesic. Consider geodesic paths $s^{i}$, such that

$$
s_{ \pm}^{i}=q_{ \pm}^{i} .
$$

If either $\left|W_{1}\right|$ or $\left|W_{2}\right|$ is larger than $4 \max \{|X|,|Y|\}$ then by Lemma 3 the paths $s^{1}$ and $s^{2}$ contain $8 \delta$-bound subsegments $t^{1}$ and $t^{2}$ such that $\left|t^{1}\right|,\left|t^{2}\right|>1 / 3\left|s^{1}\right|$ (we 


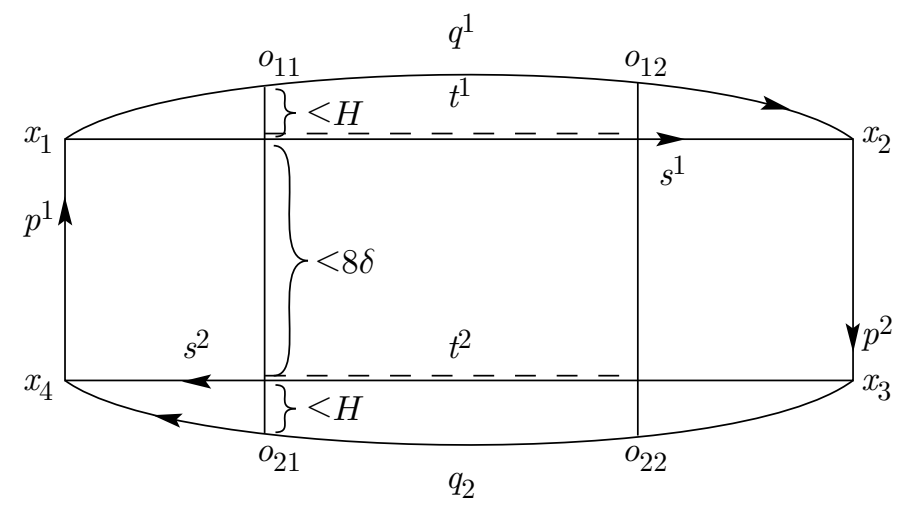

Figure 1

suppose that $\left.M_{0}>8 \delta /(7 / 20-1 / 3)\right)$. We take these subsegments to be maximal $8 \delta$-bound subsegments (this means that there are no $8 \delta$-bound subsegments $\bar{t}^{1}, \bar{t}^{2}$ such that $\bar{t}^{1}$ contains $t^{1}, \bar{t}^{2}$ contains $t^{2}$ and one of these inclusions is proper).

Lemma 2 allows us to find phase points $o_{1 j}(j=1,2)$ on $q^{1}$, and $o_{2 j}(j=1,2)$ on $q^{2}$, such that

$$
\left|o_{11}-t_{-}^{1}\right|,\left|o_{12}-t_{+}^{1}\right|,\left|o_{21}-t_{-}^{2}\right|,\left|o_{22}-t_{+}^{2}\right|<H_{2} .
$$

This shows that in the case $W_{1}=v, W_{2}=\bar{v}$ (the third case) we have either the first possibility or the third (we will show that the second possibility is a particular case of the third). The subpath $z^{1}=o_{11}-o_{12}$ has length

$$
\left|z^{1}\right|>1 / 3\left|q^{1}\right|
$$

and by Lemma 1 every vertex of $z^{1}$ can be connected with a vertex of the path $z^{2}=o_{21}-o_{22}$ by some path $t$ of length $<4 H_{2}+16 \delta$.

Consider now vertices $a_{1}, a_{2}, a_{3} \ldots$ (called phase vertices) of the path $z^{1}$, such that the labels of the subpaths $a_{i}-a_{i+1}$ are graphically equal to generators of $\mathcal{U}(\mathcal{V})$. Similarly, choose phase vertices $b_{1}, b_{2}, b_{3}, \ldots$ on $z^{2}$. As was noticed above, each $a_{i}$ can be connected with some vertex $b_{k}$ by a path $t_{i}$ of length $<2 H$. Therefore the number of different labels $\phi\left(t_{i}\right)$ of paths $t_{i}$ is less then $2|J|^{2 H}$. So, for sufficiently large $M_{0}$ there exist vertices $a_{i}$ and $a_{j}$ such that $\phi\left(t_{i}\right) \equiv \phi\left(t_{j}\right)=T$.

Let $z_{1}$ be the subpath of $z^{1}$ connecting $a_{i}$ with $a_{j}$, and $z_{2}$ be the subpath of $z^{2}$ connecting $b_{k}$ with $b_{l}$. Observe that $z_{1}$ (resp. $z_{2}$ ) can be made arbitrary long if one takes long $z^{1}$ (resp. $z^{2}$ ).

The label of the closed path $t_{i}^{-1}\left(a_{i}-a_{j}\right) t_{j}\left(b_{l}-b_{k}\right)$ is the word $T^{-1} \phi\left(z_{1}\right) T\left(\phi\left(z_{2}\right)\right)^{-1}$. If $W_{1}, W_{2} \in \mathcal{U}$ then $\phi\left(z_{1}\right), \phi\left(z_{2}\right) \in \mathcal{U}$, and if $q^{1}$ (and hence $s^{1}$ ) is long enough (increase $M_{0}$ if necessary), then because $\mathcal{U}$ is conjugate separated, one can find a suitable $T$ in $\mathcal{U}$. Hence $X, Y \in \mathcal{U}$.

If $W_{1} \in \mathcal{U}$ and $W_{2} \in \mathcal{V}$ then, because $\mathcal{H}$ is a separated HNN-extension, such a $T$ cannot exist for long $q_{1}$ or $q_{2}$.

A direct consequence of this lemma is the following

Corollary 5. Let $\mathcal{U}$ and $\mathcal{V}$ be subgroups of $\mathcal{G}$ as in Theorem 1. If

$$
M>\max \left\{M_{0}, 4, c \lambda\right\}
$$


then for any $\mathcal{U}$-geodesic words $u, \bar{u}$, any $\mathcal{V}$-geodesic words $v, \bar{v}$, and geodesic words $X, Y \in \mathcal{G}$,

- the equality $X u Y \bar{u}=1$ implies

$$
M \cdot \max (|X|,|Y|)>|u|,|\bar{u}|,
$$

or $X$ and $Y$ belong to $\mathcal{U}$.

- the equality $X u Y v=1$ implies

$$
\max (|u|,|v|)<M \max (|X|,|Y|, 1),
$$

- the equality $X v Y \bar{v}=1$ implies one of the following:

1. $M \cdot \max (|X|,|Y|)>|v|,|\bar{v}|$,

2. $X$ and $Y$ belong to $\mathcal{V}$,

3. there are elements $T_{1}$ and $T_{2}$ such that $\left|T_{i}\right|<H, v \equiv v_{1} v_{3} v_{2}, \bar{v} \equiv \bar{v}_{2} \bar{v}_{3} \bar{v}_{1}$,

$$
\begin{gathered}
X v_{1} T_{1}^{-1} \bar{v}_{1}=1, T_{1} v_{3} T_{2}^{-1} \bar{v}_{3}=1, T_{2} v_{2} Y \bar{v}_{2}=1 \\
\text { and }\left|v_{1}\right|,\left|\bar{v}_{1}\right| \leq 4 \max \{|X|, H\},\left|v_{2}\right|,\left|\bar{v}_{2}\right| \leq 4 \max \{|Y|, H\} .
\end{gathered}
$$

Definition 2. Suppose a word $W$ in $\mathcal{G}$ has the following decomposition:

$$
W \equiv X_{0} W_{1} X_{1} W_{2} X_{2} \ldots W_{k} X_{k}
$$

where each $X_{i}$ is a reduced word, each $W_{i}$ either belongs to $\mathcal{U}$ and is $\mathcal{U}$-geodesic or belongs to $\mathcal{V}$ and is $\mathcal{V}$-geodesic, and if, for some $i, W_{i}$ and $W_{i+1}$ both belong to $\mathcal{U}$ or both belong to $\mathcal{V}$ then $X_{i} \neq 1$. Then this decomposition will be called a $\mathcal{U V}$-decomposition of $W$.

If $W=1, X_{0} \equiv 1$ and in the above definition indices are taken modulo $k$, then the above decomposition will be called a cyclic $\mathcal{U} \mathcal{V}$-decomposition of $W$.

In the case where all the $W_{i}$ 's belong to $\mathcal{U}$ (resp. $\left.\mathcal{V}\right)$, we will talk about $\mathcal{U}$ decomposition $(\mathcal{V}$-decomposition).

Definition 3. A $\mathcal{U} \mathcal{V}$-decomposition of the word $W$

$$
W \equiv W_{1} X_{1} W_{2} X_{2} \ldots W_{k} X_{k}=1
$$

is called splittable if one of the following holds.

1. There is a $j \leq k$ such that $W_{1} \equiv W_{11} W_{12}, W_{11}, W_{12} \in \mathcal{U}$ and $W_{j} \equiv W_{j 1} W_{j 2}$, $W_{j 1}, W_{j 2} \in \mathcal{U}$ and

$$
W_{12} X_{1} W_{2} X_{2} \ldots W_{j 1}=W^{\prime} \in \mathcal{U}
$$

where

$$
\left|W_{12}\right|_{\mathcal{U}}+\left|W_{j 1}\right|_{\mathcal{U}}>\left|W^{\prime}\right|_{\mathcal{U}}+\left|W_{j 1} W^{\prime-1} W_{12}\right|_{\mathcal{U}}
$$

2. There is a $j \leq k$ such that $W_{1} \equiv W_{11} W_{12}, W_{11}, W_{12} \in \mathcal{V}$ and $W_{j} \equiv W_{j 1} W_{j 2}$, $W_{j 1}, W_{j 2} \in \mathcal{V}$ and

$$
W_{12} X_{1} W_{2} X_{2} \ldots W_{j 1}=W^{\prime} \in \mathcal{V}
$$

where

$$
\left|W_{12}\right|_{\mathcal{V}}+\left|W_{j 1}\right|_{\mathcal{V}}>\left|W^{\prime}\right|_{\mathcal{V}}+\left|W_{j 1} W^{\prime-1} W_{12}\right|_{\mathcal{V}}
$$

3. There is a $j \leq k$ such that $W_{1} \equiv W_{11} W_{12}, W_{11} \neq 1, W_{12} \neq 1 \in \mathcal{V}$ and $W_{j} \equiv W_{j 1} W_{j 2}, W_{j 1} \neq 1, W_{j 2} \neq 1 \in \mathcal{V}$ and

$$
W_{12} X_{1} W_{2} X_{2} \ldots W_{j 1}=T,
$$

where $T \notin \mathcal{V},|T|<H$ and $W_{12}, W_{j 1}$ have minimal length in $\mathcal{V}$ among the subwords of $W_{1}$ and $W_{j}$ with the property that the above equality is satisfied for some word $T$, where $|T|<H$. 
A cyclic $\mathcal{U V}$-decomposition of $W$ is called cyclically nonsplittable if all permutations of the form

$$
W_{i} X_{i} \ldots W_{k} X_{k} W_{1} \ldots W_{i-1} X_{i-1}=1 .
$$

are nonsplittable.

Our main goal in this section is to prove Corollary 6 .

The following lemma follows from Corollary 5 .

Lemma 5. For any $\mathcal{U}$-geodesic words $u, \bar{u} \in \mathcal{U}$, and $\mathcal{V}$-geodesic words $v, \bar{v} \in \mathcal{V}$ and geodesic words $X, Y \in \mathcal{G}$ we have the following:

- If $X u Y \bar{u}=1$ is a cyclically nonsplittable $\mathcal{U}$-decomposition, then

$$
M \cdot(|X|+|Y|)>|u|,|\bar{u}| .
$$

- The equality $X u Y v=1$ implies

$$
\max (|u|,|v|)<M \max (|X|+|Y|, 1) .
$$

- If $X v Y \bar{v}=1$ is a cyclically nonsplittable $\mathcal{V}$-decomposition, then

$$
M \cdot(|X|+|Y|)>|v|,|\bar{v}| .
$$

Proposition 1. Suppose that in the group $\mathcal{G}$ we have a cyclically nonsplittable $\mathcal{U} \mathcal{V}$ decomposition of the word $W$ :

$$
W \equiv W_{1} X_{1} W_{2} X_{2} \ldots W_{k} X_{k}=1,
$$

where the $X_{i}$ are geodesic words.

Then for any $i$

$$
\left|W_{i}\right| \leq M\left(\sum_{i=1}^{k}\left|X_{i}\right|\right)+2 M(k-1)\left(2 H_{2}+6 \delta\right)+k\left(H_{2}+1\right) .
$$

(See Fig. 3.)

We will give a proof of this proposition together with the following lemma by simultaneous induction on $k$.

Lemma 6. Suppose we are given a $\mathcal{U} \mathcal{V}$-decomposition of a word $\bar{W}$ :

$$
\bar{W} \equiv \bar{X}_{0} \bar{W}_{1} \bar{X}_{1} \bar{W}_{2} \bar{X}_{2} \ldots \bar{W}_{k-2} \bar{X}_{k-2} \bar{W}_{k-1}
$$

$k>2$, and $\bar{X}_{0}=1$ if $k>3$, the $\bar{X}_{i}$ 's are geodesic, each $\bar{W}_{i}$ either belongs to $\mathcal{U}$ and is geodesic in $\mathcal{U}$, or belongs to $\mathcal{V}$ and is geodesic in $\mathcal{V}$.

Let $U_{k}$ be a geodesic word such that $U_{k}=\bar{W}$ and $\bar{U}_{k}$ be a geodesic such that

$$
\bar{U}_{k}=U_{k} \bar{W}_{k-1}^{-1} .
$$

Suppose that the decomposition

$$
\bar{X}_{0} \bar{W}_{1} \bar{X}_{1} \bar{W}_{2} \bar{X}_{2} \ldots \bar{W}_{k-2} \bar{X}_{k-2} \bar{W}_{k-1} U_{k}^{-1}=1
$$

is cyclically nonsplittable. Let $\bar{V}_{i}$ be a geodesic word such that $\bar{V}_{i}=\bar{W}_{i}$. Then Gromov's products

$L_{k}=\left(\bar{U}_{k}^{-1} \cdot \bar{V}_{k-1}\right) \leq M\left(\sum_{i=1}^{k-2}\left|\bar{X}_{i}\right|\right)+M(1+2(k-2))\left(2 H_{2}+6 \delta\right)+(k-1)\left(H_{2}+1\right)$.

(See Figs. 2, 4.) 


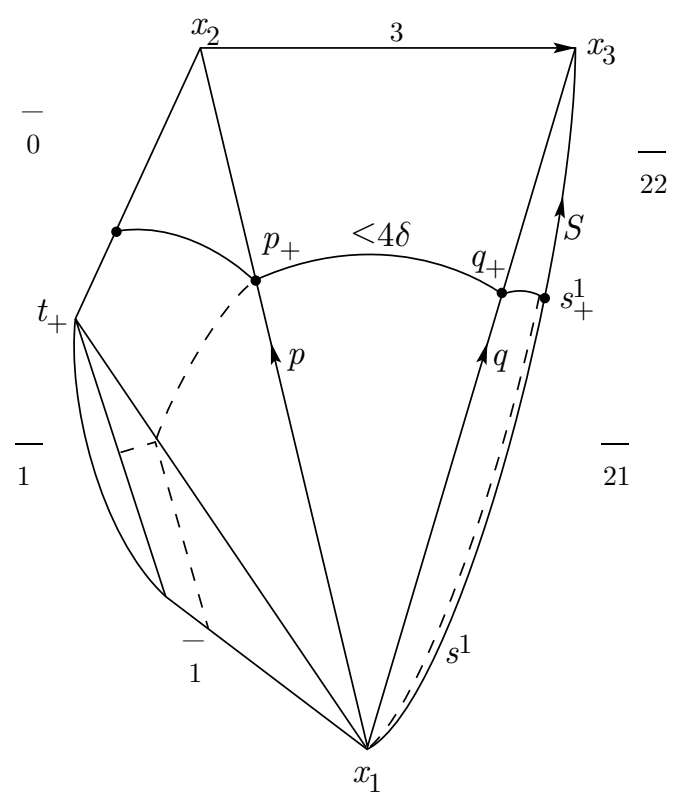

Figure 2

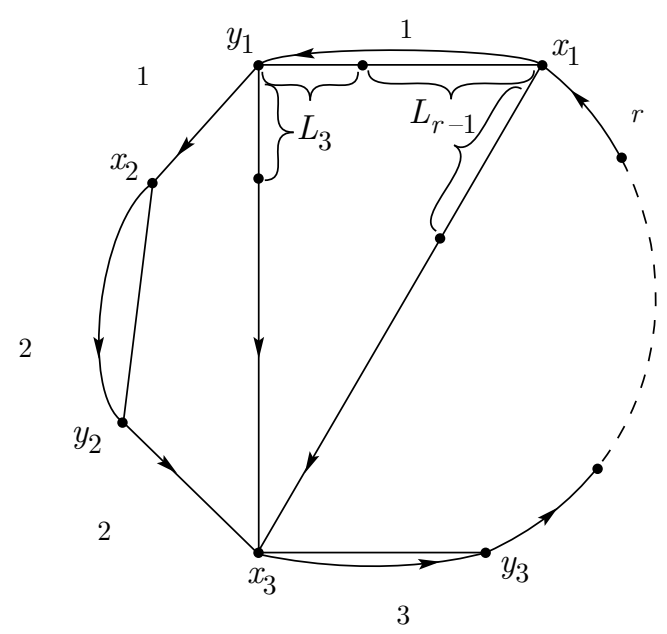

FiguRE 3

The assertion of the proposition for $k=2$ is just Lemma 5 .

We first will prove Lemma 6 for $k=3$. The proof is illustrated in Fig. 2. Consider a geodesic triangle $\left[x_{1}, x_{2}, x_{3}\right]$ in $\Gamma(\mathcal{G})$ such that the label $\bar{A}$ of the side $\left[x_{1}, x_{2}\right]$ is equal to $\bar{U}_{3}^{-1}$ in $\mathcal{G}$ and the label $\bar{B}$ of the side $\left[x_{1}, x_{3}\right]$ is equal to $\bar{V}_{2}$. Then $L_{3}=(\bar{A} \cdot \bar{B})$. By Lemma 1, one can find decompositions $\bar{A} \equiv A_{1} A_{2}, \quad \bar{B} \equiv B_{1} B_{2}$, where $\left|A_{1}\right|=\left|B_{1}\right|>L_{3}-1$, and for subsegments $p, q$ of the sides $\left[x_{1}, x_{2}\right]$ and $\left[x_{1}, x_{3}\right]$ with labels $A_{1}$ and $B_{1}, \quad\left|p_{+}-q_{+}\right| \leq 4 \delta$. Consider the path $s$ issuing from $x_{1}$ and having $\bar{W}_{2}$ as a label. According to Lemma 2 there is an initial subpath $s^{1}$ 


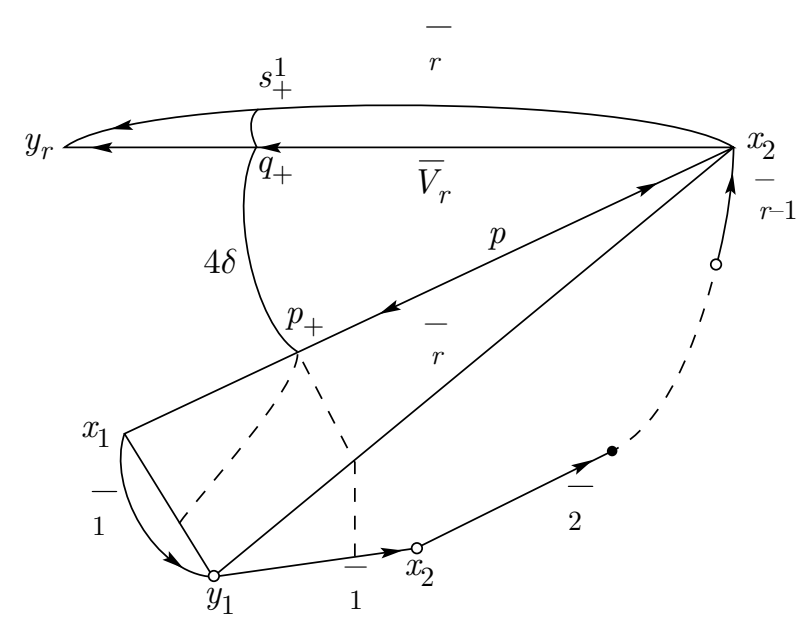

FiguRE 4

of $s$ with the label $\bar{W}_{21}$ belonging to the same subgroup $\mathcal{U}$ or $\mathcal{V}$ as $W_{2}$, such that $\left|q_{+}-s_{+}^{1}\right|<H_{1}+c=H_{2}$. Let $t$ be a path issuing from $x_{1}$ and having the label $\bar{X}_{1}^{-1} \bar{W}_{1}^{-1}$. Then either $\left|t_{+}-p_{+}\right|<\delta+\left|\bar{X}_{0}\right|$ or there is a subpath $t^{1}$ issuing from $x_{1}$ and having the label $\bar{X}_{1}^{-1} \bar{W}_{12}^{-1}$, such that $\left|t_{+}^{1}-p_{+}\right|<2 \delta+H_{2}$.

It is clear that $L_{3}-1 \leq H_{2}+\left|\bar{W}_{21}\right|$.

By Corollary $5,\left|\bar{W}_{21}\right|<M \max \left\{\left|\bar{X}_{1}\right|, H_{2}+5 \delta+\left|\bar{X}_{0}\right|, 2 H_{2}+6 \delta, H_{2}+6 \delta+\left|\bar{X}_{1}\right|, 1\right\}$. Hence $L_{3} \leq H_{2}+1+M\left(\left(\left|\bar{X}_{0}\right|+\left|\bar{X}_{1}\right|\right)+2 H_{2}+6 \delta\right)$. The lemma is proved for $k=3$.

Suppose now that the proposition is proved for all $k<r$ and the lemma is proved for all $k \leq r$. We will prove the proposition for $k=r$. Let $V_{i}$ be geodesic words such that $V_{i}=W_{i}$. See Fig. 3. Let $\left[x_{1}, y_{1}, \ldots x_{r}, y_{r}\right]$ be a geodesic $2 r$-gon in the Cayley graph $\Gamma(\mathcal{G})$, such that $V_{i}$ is the label of $\left[x_{i}, y_{i}\right]$ and $X_{i}$ is the label of $\left[y_{i}, x_{i+1}\right]\left(X_{r}\right.$ is the label of $\left.\left[y_{r}, x_{1}\right]\right)$. Let $U$ be the label of $\left[y_{1}, x_{3}\right]$, and $W$ the label of $\left[x_{1}, x_{3}\right]$.

By the assertion of the lemma for $k=r$, applied to the word

$$
W_{3} X_{3} \ldots W_{r} X_{r} W_{1} \text {, }
$$

we have

$$
L_{r}=\left(W \cdot V_{1}\right) \leq(r-1)\left(H_{2}+1\right)+M\left(\sum_{i=3}^{r}\left|X_{i}\right|+(1+2(r-2))\left(2 H_{2}+6 \delta\right)\right) .
$$

By the assertion of the Lemma for $k=3$, applied to the word

$$
X_{2}^{-1} W_{2}^{-1} X_{1}^{-1} W_{1}^{-1} \text {, }
$$

we have

$$
L_{3}=\left(U \cdot V_{1}^{-1}\right) \leq H_{2}+1+M\left(\left(\left|X_{0}\right|+\left|X_{1}\right|\right)+2 H_{2}+6 \delta\right) .
$$

Now, by Lemma 1 ,

$$
\left|V_{1}\right|=L_{3}+L_{r} \leq r\left(H_{2}+1\right)+M\left(\sum_{i=1}^{r}\left|X_{i}\right|+(r-1) 2\left(2 H_{2}+6 \delta\right)\right) .
$$

Now we will prove the lemma for $k=r+1$. See Fig. 4 . 
Consider a $2 r$-gon $\left[x_{1}, y_{1}, \ldots, x_{r}, y_{r}\right]$ in $\Gamma(\mathcal{G})$ such that $\bar{X}_{i}$ is the label of $\left[y_{i}, x_{i+1}\right]$ and a path with the label $\bar{W}_{i}$ has initial point $x_{i}$ and terminal point $y_{i}$.

Consider a geodesic triangle $\left[x_{1}, x_{r}, y_{r}\right]$ such that the label $\bar{A}$ of the side $\left[x_{r}, x_{1}\right]$ is equal to $\bar{U}_{r}^{-1}$ in $\mathcal{G}$, and the label $\bar{B}$ of the side $\left[x_{r}, y_{r}\right]$ is equal to $\bar{V}_{r}$. Then $L_{r}=(\bar{A} \cdot \bar{B})$. By Lemma 1, one can find decompositions $\bar{A} \equiv A_{1} A_{2}, \quad \bar{B} \equiv B_{1} B_{2}$, where $\left|A_{1}\right|=\left|B_{1}\right|>L_{r}-1$ and for subsegments $p, q$ of the sides $\left[x_{r}, x_{1}\right]$ and $\left[x_{r}, y_{r}\right]$ with labels $A_{1}$ and $B_{1}, \quad\left|p_{+}-q_{+}\right| \leq 4 \delta$. Consider the path $s$ issuing from $x_{r}$ and having $\bar{W}_{r}$ as its label. According to Lemma 2 there is an initial subpath $s^{1}$ of $s$ with the label $\bar{W}_{r 1}$ belonging to the same subgroup $\mathcal{U}$ or $\mathcal{V}$ as $W_{r}$, such that $\left|q_{+}-s_{+}^{1}\right|<H_{2}$. There are the following $r$ possibilities: $\left|p_{+},\left[x_{1}, y_{1}\right]\right|<\delta$, $\left|p_{+}, x_{i+1}\right|<i \delta+\left|\bar{X}_{i}\right|(i=1, \ldots, r-1)$.

We consider the first possibility. Then there is a path $t$ issuing from $y_{1}$ with the label $\bar{W}_{12}^{-1}$ such that $\left|p_{+}, t_{+}\right|<\delta+H_{2}$. Let $f$ be the label of the geodesic path $s_{+}^{1}, t_{+} ;$then $|f|<5 \delta+2 H_{2}$.

If $f \neq 1$ or both $\bar{W}_{12}, \bar{W}_{r 1} \notin \mathcal{U}(\mathcal{V})$, then the word

$$
\bar{W}_{12} \bar{X}_{1} \ldots \bar{W}_{r-1} \bar{X}_{r-1} \bar{W}_{r 1} f=1
$$

is nonsplittable and we can apply the proposition for $k \leq r$ to get

$$
L_{r+1}-1 \leq r\left(H_{2}+1\right)+M\left(\sum_{i=1}^{r-1}\left|\bar{X}_{i}\right|+1+2(r-1)\left(2 H_{2}+6 \delta\right)\right) .
$$

Now suppose that $f=1, \bar{W}_{12}, \bar{W}_{r 1} \in \mathcal{U}$. Let $\hat{W}$ be a quasigeodesic word such that $\hat{W}=\bar{W}_{r 1} \bar{W}_{12}$. Consider instead of the word

$$
\bar{W}_{12} \bar{X}_{1} \ldots \bar{W}_{r-1} \bar{X}_{r-1} \bar{W}_{r 1}=1
$$

the word

$$
\hat{W} \bar{X}_{1} \ldots \bar{W}_{r-1} \bar{X}_{r-1}
$$

(it is nonsplittable), and apply the proposition for $k=r-1$ to estimate $\hat{W}$.

Because nonsplitability implies $\left|\left(\bar{W}_{r 1} \bar{W}_{12}\right)\right|_{\mathcal{U}}=\left|\bar{W}_{r 1}\right|_{\mathcal{U}}+\left|\bar{W}_{12}\right|_{\mathcal{U}}$, and hence $\left|\bar{W}_{r 1}\right| \leq|\hat{W}|+H_{2}$, the proof is finished in this case.

The case $f=1, \bar{W}_{12}, \bar{W}_{r 1} \in \mathcal{V}$ can be considered similarly.

The other $r-1$ possibilities $\left|p_{+}, x_{i+1}\right|<i \delta+\left|\bar{X}_{i}\right|(i=1, \ldots r-1)$ can be considered similarly to the first possibility. Proposition 1 and Lemma 6 are proven.

Corollary 6. There are constants $M_{1}, M_{2}$ such that if in the group $\mathcal{G}$ we have a cyclically nonsplittable $\mathcal{U} \mathcal{V}$-decomposition

$$
W \equiv W_{1} X_{1} W_{2} X_{2} \ldots W_{k} X_{k}=1,
$$

then for $W_{i} \in \mathcal{U}$ we have $\left|W_{i}\right|_{\mathcal{U}} \leq M_{1} \sum_{i}\left|X_{i}\right|+M_{2} k$, and for $W_{i} \in \mathcal{V}$ we have $\left|W_{i}\right|_{\mathcal{V}} \leq M_{1} \sum_{i}\left|X_{i}\right|+M_{2} k$

\section{Diagrams}

Recall that a map a is finite, planar connected 2-complex.

By a diagram $\Delta$ over a presentation $\left\langle a_{1}, \ldots, a_{m} \mid R_{1}, \ldots, R_{n}\right\rangle$, where the words $R_{i}$ are cyclically reduced, we mean a map with a function $\phi$ which assigns to each edge of the map one of the letters $a_{k}^{ \pm 1}, 1 \leq k \leq m$, such that $\phi\left(e^{-1}\right)=(\phi(e))^{-1}$ and if $p=e_{1} \ldots e_{d}$ is the contour of some cell $\Phi$ of $\Delta$, then $\phi(p) \equiv \phi\left(e_{1}\right) \ldots \phi\left(e_{d}\right) \equiv R$ 
is a cyclic shift of one of the defining words $R_{i}^{ \pm 1}$. In general the word $\phi(p)$ is called the label of the path $p$. The label of a diagram $\Delta$ (whose contour is always taken with a counterclockwise orientation) is defined analogously.

Van Kampen's lemma states that a word $W$ represents the identity of the group $G$ if and only if there is a simply connected (or Van Kampen, or disk) diagram $\Delta$ over $G$ such that the boundary label of $\Delta$ is $W$.

Due to Van Kampen's lemma, a group $G$ is hyperbolic if and only if there are constants $K$ and $C$ such that for any element $W=1$ in $\mathcal{G}$ there is a diagram with boundary label $W$ and number of cells $\leq K\|W\|+C$. Since $\|W\| \geq 1$ we can assume (taking $K+C$ instead of $K$ ) that $C=0$.

\section{HNN-EXTENSIONS}

Let $\mathcal{H}$ be the HNN-extension as in Theorem 1. We fixed the presentation for the group $\mathcal{H}$ in the first section. As we just noticed, from the hyperbolicity of the group $\mathcal{G}$ it follows that there is a constant $K$ such that for any element $W=1$ in $\mathcal{G}$ there is a diagram with boundary label $W$ and number of cells $\leq K\|W\|$.

The contents of this section is the proof of the following fact.

Proposition 2. There is a linear function $L(x)$ depending only on $\mathcal{G}, \mathcal{U}, \mathcal{V}$ such that for any element $W=1$ in $\mathcal{H}$ there is a diagram over $\mathcal{H}$ with boundary label $W$ and number of cells $\leq L(\|W\|)$.

Let $\Delta$ be a diagram over $\mathcal{H}$ with boundary label $W$. New cells corresponding to the relations $t^{-1} a_{i} t=b_{i}$, where $i=1, \ldots, n$, will be called $t$-cells. They are shown on Fig. 5. A configuration of $t$-cells in a diagram over $\mathcal{H}$, as shown on Fig. 6a, we call a $t$-annulus.

From now on we suppose that $\Delta$ is minimal; this means that it has a minimal possible number of $t$-cells.

Lemma 7. A minimal diagram over $\mathcal{H}$ cannot contain a $t$-annulus.

Proof. Suppose it contains a $t$-annulus. Take a $t$-annulus such that there is not another $t$-annulus inside it. Then the label of its internal contour $p$ equals the identity in the group $\mathcal{G}$. Hence the label of its external contour $q$ equals the identity in the group $\mathcal{G}$. We can decrease the number of $t$-cells by applying

Transformation 1. Assuming that the contour $p$ in Fig. 6a bounds a $\mathcal{G}$-diagram, replace the interior of the diagram having the contour $q$ in Fig. 6a by a $\mathcal{G}$-diagram with the contour $q$.

The lemma is proved.

By this lemma, $t$-cells can only form $t$-strips as shown in Fig. $6 \mathrm{~b}$, and these $t$-strips must end on the boundary of $\Delta$.

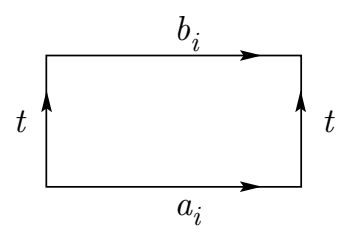

FiguRE 5 
(a)

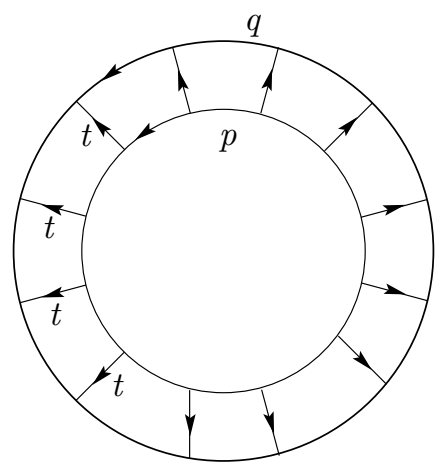

(b)

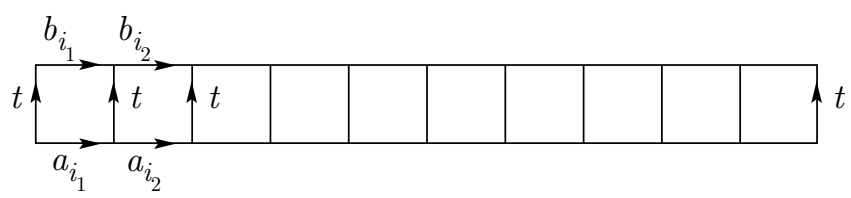

FiguRE 6

The direction of the $t$-edges defines $\mathcal{U}$-and $\mathcal{V}$-sides of a $t$-strip. The minimality of $\Delta$ also implies that the $\mathcal{U}$-sides $(\mathcal{V}$-sides) of the $t$-strips are geodesic words respectively in the subgroups $\mathcal{U}$ and $\mathcal{V}$. Indeed, suppose we have a $t$-strip whose $\mathcal{U}$ - and $\mathcal{V}$-sides are not geodesic words in $\mathcal{U}$ and $\mathcal{V}$. Let the path $p$ correspond to the $\mathcal{V}$-side of the $t$-strip, with $\phi(p)=V_{1}$. Let $V_{2}$ be a geodesic word in $\mathcal{V}$ such that $V_{2}=V_{1}$ in $\mathcal{V}$. Let $V_{2}=\phi(q)$. We replace the $t$-strip by the diagram $\Theta$ having the same contour as shown in Fig. 7 and cut out the annulus. As a result we have a diagram with fewer $t$-cells.

The typical form of $\Delta$ is shown in Fig. 8 on page 000 .

Our diagram $\Delta$ is subdivided by the $t$-strips into a set of mutually disjoint maximal $\mathcal{G}$-subdiagrams. The maximal $\mathcal{G}$-subdiagrams are the connected components of $\Delta$ which remain after deleting all $t$-edges and interiors of $t$-cells.

Our next goal is to study maximal $\mathcal{G}$-subdiagrams in the diagram $\Delta$. A typical form of a maximal $\mathcal{G}$-subdiagram is shown in Fig. 9 on page 000.

Definition 4. An island in a maximal $\mathcal{G}$-subdiagram is a $\mathcal{G}$-subdiagram with the following properties:

1. The contour of the island is subdivided into paths, such that every path either belongs to the boundary of $\Delta$ or is a part of a $\mathcal{U}$ - or $\mathcal{V}$-side of a $t$-strip in $\Delta$.

2. There is no point on the contour of the island such that the deletion of this point splits the island into two or more disconnected components.

Every edge of an island is proper, i.e. lies on the boundary of some cell of this island.

A bridge is a pair of paths $\left\{p, p^{-1}\right\}$, where $p=e_{1} \ldots e_{r}$ is a maximal subpath, consisting of improper edges of $\Delta$, such that the valencies of the terminal points of $e_{1} \ldots e_{r-1}$ are equal to 2 .

Every maximal $\mathcal{G}$-subdiagram consists of islands and bridges. 

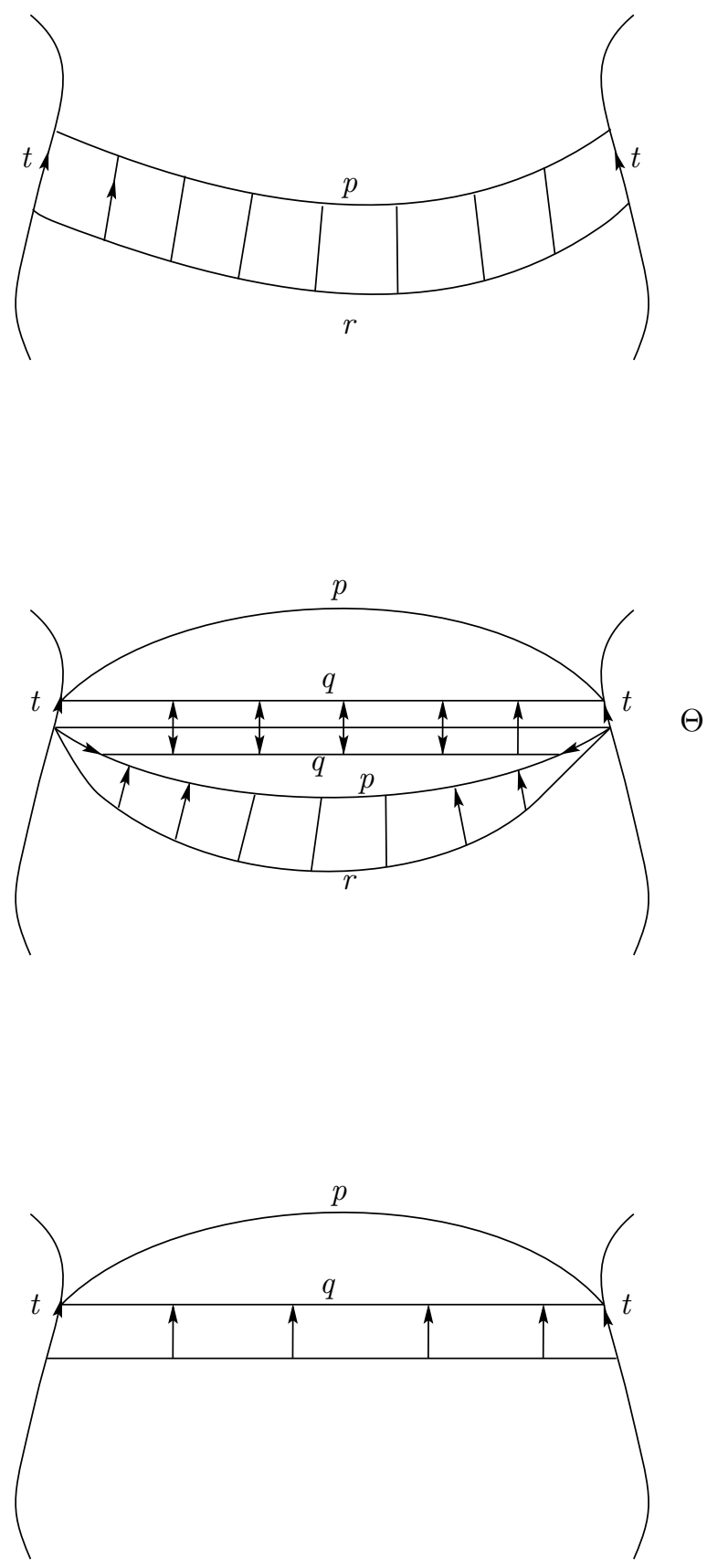

FIGURE 7

The contour of each island is canonically subdivided into paths, and each of these paths either belongs to the $\mathcal{U}$ - or $\mathcal{V}$-side of some $t$-strip and is maximal with respect to this property (we will call these paths $\mathcal{U}$-paths and $\mathcal{V}$-paths, or generally 


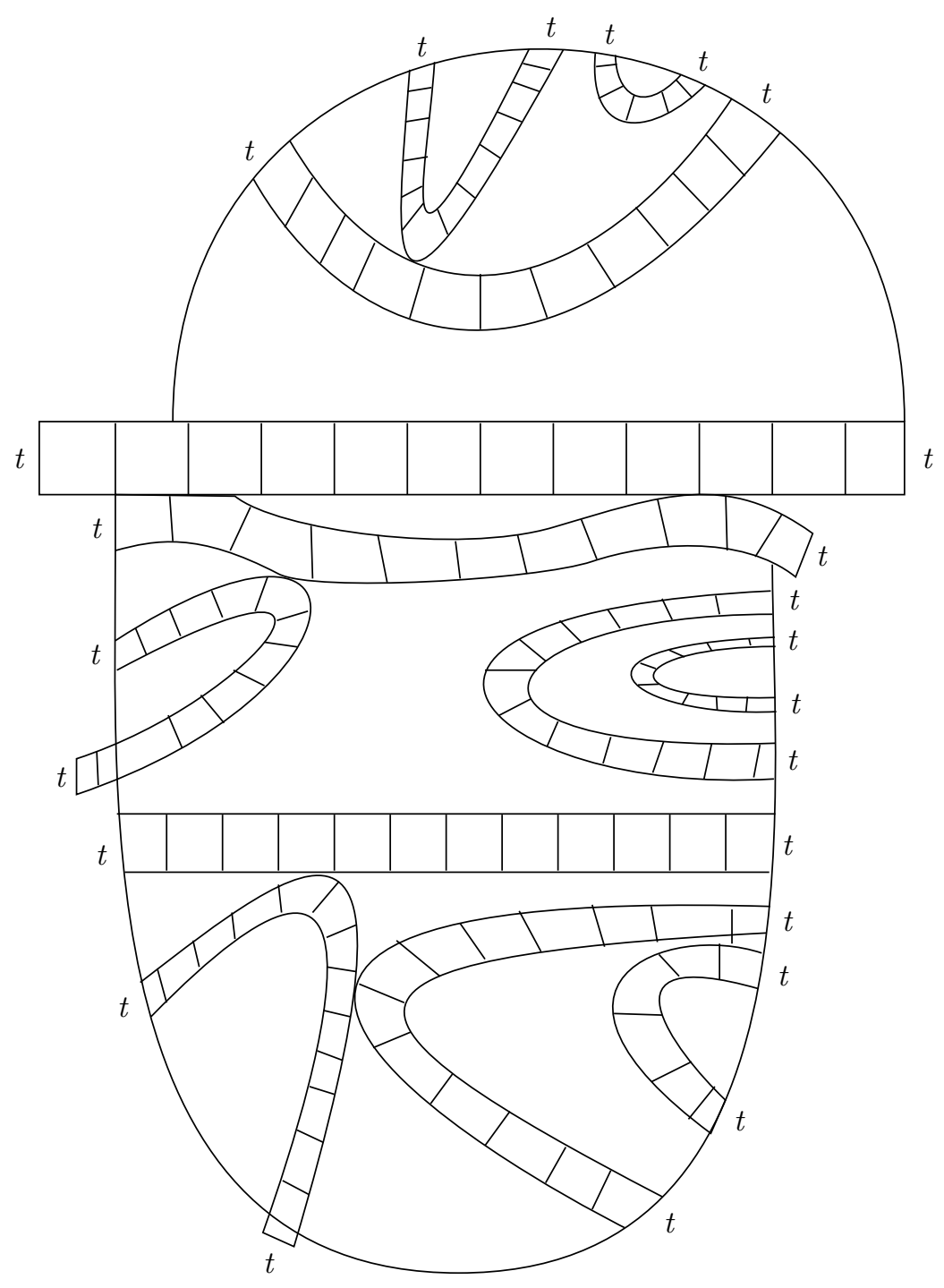

Figure 8

strippaths) or belongs to the boundary of $\Delta$ and is maximal with respect to this property (we will call them boundary paths).

We will call a vertex on a $\mathcal{U}$-side (resp. $\mathcal{V}$-side) of a $t$-strip a phase vertex if it corresponds to the beginning or end of a path labelled by some generator $a_{i}$ of $\mathcal{U}$ (resp. generator $b_{i}$ of $\mathcal{V}$ ) and to the beginning or to the end of the $t$-edge. A vertex on a strippath will be called a phase vertex if it is a phase vertex on the corresponding $\mathcal{U}$ - or $\mathcal{V}$-side of the $t$-strip.

Transformation 2. We now carry out surgeries on the diagram. Our objective is to make $\mathcal{U}$ - and $\mathcal{V}$-paths contact the boundary paths only through phase vertices. See Fig. 10. Let $p$ be a boundary-path of an island $I$ which is adjacent to a $\mathcal{U}$-path at the point $O_{1}$. Let $O_{2}$ be the phase vertex on the $\mathcal{U}$-path closest to $O_{1}$, and $q$ 


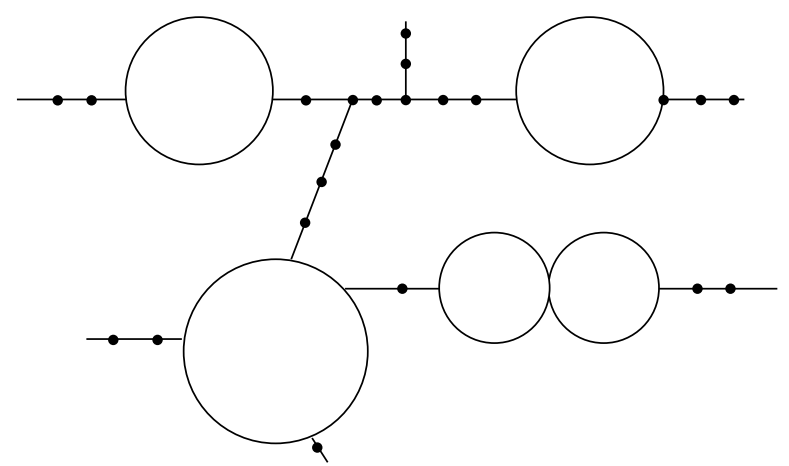

FIGURE 9
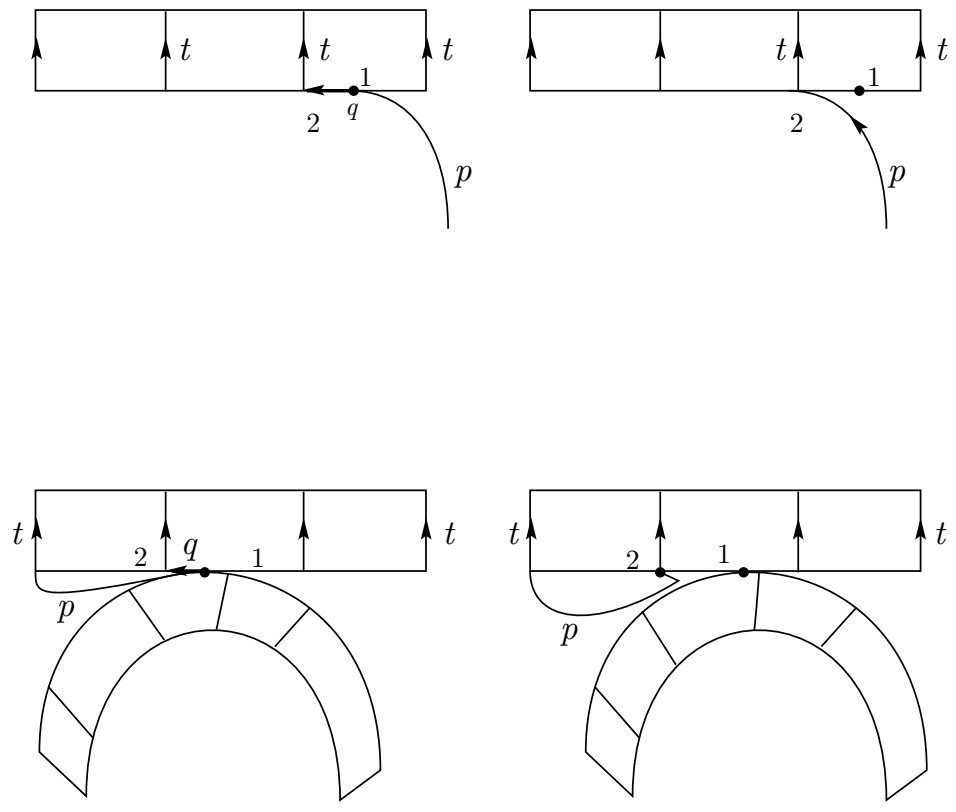

FIGURE 10

a subpath of the $\mathcal{U}$-path connecting $O_{1}$ and $O_{2}$. We make a cut along the path $q$. Each cut can increase the boundary by most $2 c$. Collectively, these cuts define Transformation 2.

Such a transformation increases the length of the boundary of $\Delta$ by a factor of not more then $(1+4 c)$. This coefficient does not depend on the diagram. So, if we can prove a linear isoperimetric unequality for a transformed diagram (or diagrams), we can prove it for the original one.

Without loss of generality we assume now that $\Delta$ has the property that all boundary paths contact strip-paths only at phase vertices.

Transformation 3. For each boundary-path $q$ on the contour of an island, linking two $\mathcal{V}$-paths and such that $\phi(q)$ is equal in $\mathcal{G}$ to an element of $\mathcal{V}, \phi(q)=v$, as shown 


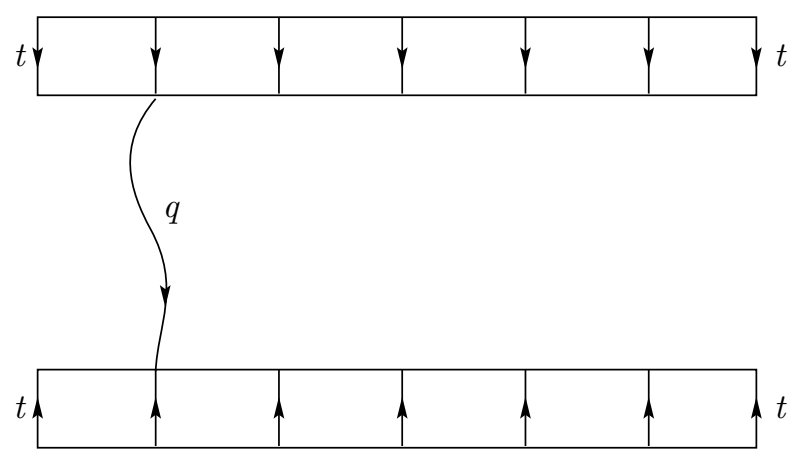

FIGURE 11

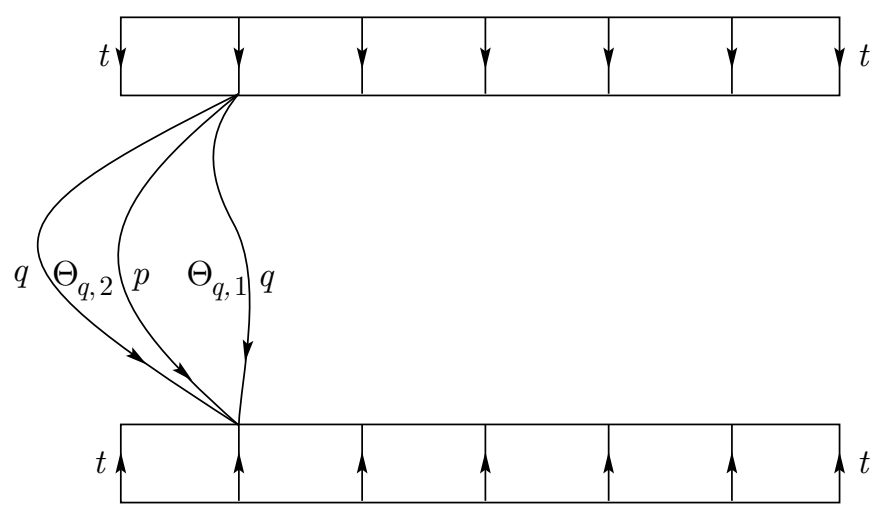

FiguRE 12

on Fig. 11, glue to $q$ two diagrams over $\mathcal{G}$ : $\Theta_{q, 1}$ with contour $q^{-1} p$ and $\Theta_{q, 2}$ with contour $p^{-1} q$, where $\phi(p) \equiv v$ and $v$ is a geodesic word in $\mathcal{V}$. See Fig. 12 .

We do the same for each boundary-path $q$ such that $\phi(q)$ is equal in $\mathcal{G}$ to an element of $\mathcal{U}$ and linking two $\mathcal{U}$-paths. The resulting diagram will have the same contour as $\Delta$. Transformation 3 ends by cutting out each diagram $\Theta_{q, 2}$.

Since $\mathcal{U}$ and $\mathcal{V}$ are quasiisometrically embedded in $\mathcal{G}$ and $\mathcal{G}$ is hyperbolic, we can pick each diagram $\Theta_{q, 2}$ over $\mathcal{G}$, with a contour $p q^{-1}$, where $\phi(p)=v(u)$, so that the number of cells in it is less than $K(\|v\|+\|q\|) \leq K(c \lambda+1)\|q\|$. Hence the sum of cells in all the diagrams $\Theta_{q, 2}$ is less than $K(c \lambda+1)\|W\|$.

Our goal is to bound the number of cells in the diagrams with contour $W$ by a linear function of $\|W\|$. We have bounded the number of cells in the union of the diagrams $\Theta_{q, 2}$ for all boundary paths $q$ of the type considered above. The length of the contour of the resulting diagram is less then $c \lambda$ times the length of the contour of the original diagram $\Delta$. Now instead of the diagram $\Delta$ we will consider this new diagram, which we will also denote by $\Delta$.

From now on we do not change the boundary of $\Delta$ anymore.

Lemma 8. If in the diagram $\Delta$ on the boundary of an island there are two $\mathcal{U}$-paths $(\mathcal{V}$-paths) $p$ and $s$ such that the terminal vertex of $p$ is the initial vertex of $s$ and is a phase vertex for both $p$ and $s$, then the path ps is a geodesic in the group $\mathcal{U}(\mathcal{V})$. 
Proof. See Fig. 13. Suppose that on the boundary of an island two $\mathcal{U}$-paths $p$ and $s$ have a common phase vertex $p_{+}=s_{-}$with $\phi(p)=u_{1}, \phi(s)=u_{2}$. Suppose that their union is not a geodesic path in $\mathcal{U}$, and let $q$ be a geodesic path in $\mathcal{U}$ such that $\phi(q)=u$ and $u=u_{1} u_{2}$. So $|q|_{\mathcal{U}}<|p|_{\mathcal{U}}+|s|_{\mathcal{U}}$.

We make the

Transformation 4 as shown on Fig. 13 (the contour of the subdiagram is not changed by this transformation). We cut along the path $p s$ and insert two mirror copies of the diagram with the contour $p s q^{-1}$. Then we cut along the edge $q$ and insert a patch of two adjoining $t$-strips. We then cut $6 t$-cells and reattach them in a different way to create the subdiagram in Fig. 13c. Transformation 4 ends by cutting out the $t$-annulus (Transformation 1 ).

After the cutting we have a diagram with fewer $t$-cells. This contradicts the minimality of $\Delta$. Indeed, we replace $|p|_{\mathcal{U}}+|s|_{\mathcal{U}} t$-cells by $|q|_{\mathcal{U}} t$-cells. This completes the proof of the lemma.

In the diagram $\Delta, \mathcal{U}$-sides of two $t$-strips cannot be glued together along a path longer than $M c+c$. If they are glued from one, common phase vertex to another one, then we can make a $t$-annulus, then cut it out using Transformation 1 and decrease the number of $t$-cells. This contradicts the minimality of $\Delta$. If they are glued not from one common phase vertex to another one, then we can apply Corollary 5 and restrict their length by $M c+c$.

Definition 5. A $\mathcal{G}$-subdiagram with a cyclic $\mathcal{U} \mathcal{V}$-decomposition of boundary label

$$
W \equiv W_{1} X_{1} W_{2} X_{2} \ldots W_{k} X_{k}=1,
$$

where each $W_{i}$ is a label of a strippath, is called nonsplittable if the above decomposition is cyclically nonsplittable (see Definition 3).

Definition 6. A maximal nonsplittable $\mathcal{G}$-subdiagram is called a nonsplittable piece.

Definition 7. A $\mathcal{V}$-piece is a $\mathcal{G}$-subdiagram having boundary label

$$
W_{1} X_{1} W_{2} X_{2}=1 \text {, }
$$

where $W_{1}, W_{2}$ label only subpaths of $\mathcal{V}$-sides of $t$-strips, and $X_{1}, X_{2}$ are shorter than $H$ (the $X_{i}$ 's can be trivial), and we assume that the $\mathcal{V}$-piece is not properly contained in another $\mathcal{G}$-subdiagram with a boundary label of that type.

Definition 8. By a piece we mean either a nonsplittable piece or a $\mathcal{V}$-piece.

A piece may consist of a several islands; it need not necessarily be an island itself.

A contour of a piece consists of strippaths (which are maximal with respect to the property of belonging to a side of a $t$-strip and to the contour of the piece, and beginning and ending in the phase vertex) and paths connecting them.

Lemma 9. Every maximal $\mathcal{G}$-subdiagram in $\Delta$ is partitioned into nonsplittable pieces and $\mathcal{V}$-pieces between them.

Proof. Let $\Theta$ be a maximal $\mathcal{G}$-subdiagram. A boundary label of $\Theta$ is a word

$$
W \equiv W_{1} X_{1} W_{2} X_{2} \ldots W_{k} X_{k}=1,
$$

where $W_{i} \in\{\mathcal{U}, \mathcal{V}\}$ and is geodesic in $\mathcal{U}$ or in $\mathcal{V}$. Also if $W_{i}, W_{i+1} \in \mathcal{U}$ or $W_{i}, W_{i+1} \in$ $\mathcal{V}$ (indices are taken modulo $k$ ), then $X_{i} \neq 1$. 
(a)

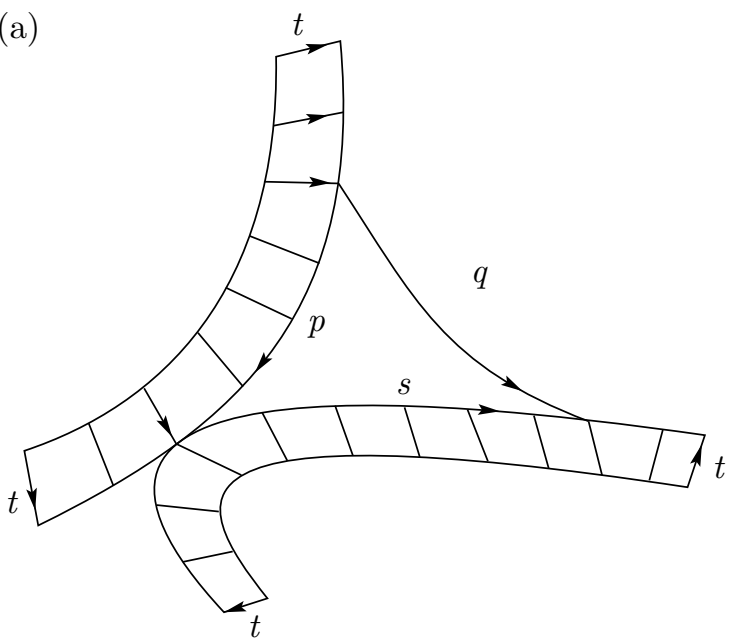

(b)

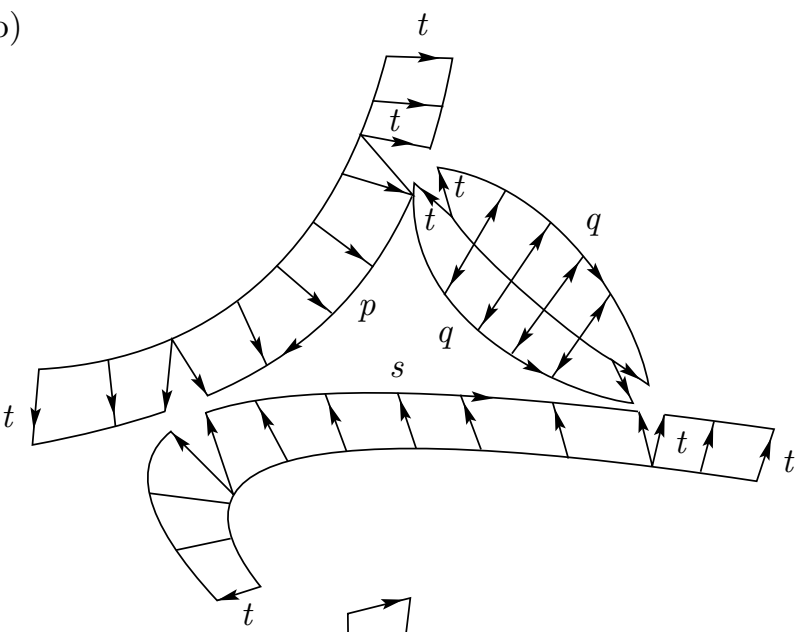

(c)

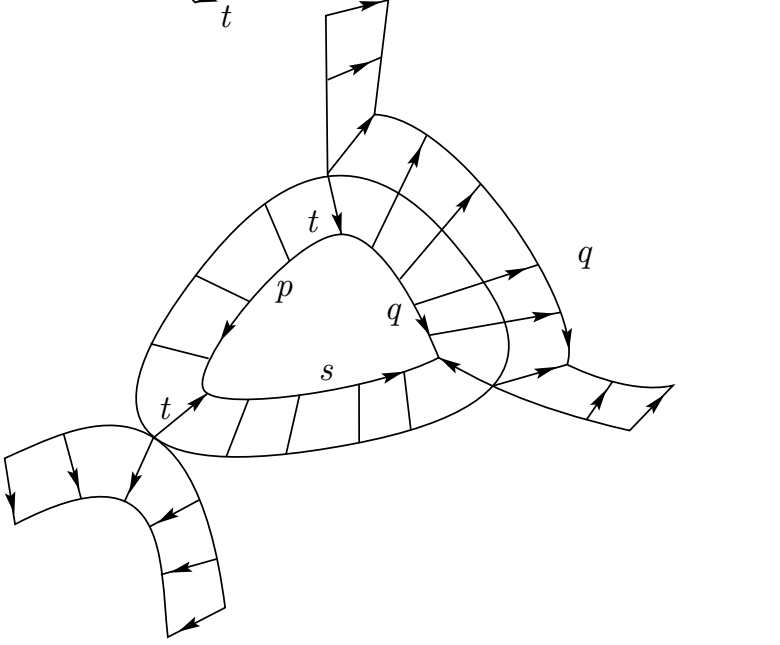

FiguRe 13 
Suppose the boundary label is a splittable $\mathcal{U} \mathcal{V}$-decomposition. Suppose that the second possibility in Definition 2 holds. Then there is a $j \leq k$ such that $W_{1} \equiv W_{11} W_{12}, W_{11}, W_{12} \in \mathcal{V}, W_{j} \equiv W_{j 1} W_{j 2}, W_{j 1}, W_{j 2} \in \mathcal{V}$ and

$$
W_{12} X_{1} W_{2} X_{2} \ldots W_{j 1}=W^{\prime} \in \mathcal{V}
$$

where

$$
\left|W_{12}\right|+\left|W_{j 1}\right|>\left|W^{\prime}\right|+\left|W_{j 1} W^{\prime-1} W_{12}\right| .
$$

Let $\bar{W}_{11}, \bar{W}_{12}, \bar{W}_{j 1}, \bar{W}_{j 2}$ be the words corresponding to $W_{11}, W_{12}, W_{j 1}, W_{j 2}$ on the other side of the $t$-strips. Then we can make

Transformation 5 as shown in Fig. 14. Let $V$ be a geodesic word in $\mathcal{V}$ such that $V=W_{j 1} W^{\prime-1} W_{12}$. Let $\bar{V}$ be the word corresponding to $V$ on the other side of the $t$-strip. Let $\Delta_{1}$ be the subdiagram with the contour

$$
t^{-1} \bar{W}_{1} t V^{-1} t^{-1} \bar{W}_{j} t W_{j 2}^{-1} W^{\prime-1} W_{11}^{-1} .
$$

Replace it by the union of three diagrams: $\Theta_{1}$, which is just a $t$-strip with the boundary label $t V^{-1} t^{-1} \bar{V}, \Theta_{2}$, with the boundary label

$$
\bar{V}^{-1} t t^{-1} \bar{W}_{j 1} t t^{-1} \bar{W}^{\prime-1} t t^{-1} \bar{W}_{12} t t^{-1}
$$

and $\Theta_{3}$, with the boundary label

$$
\bar{W}_{11} t t^{-1} \bar{W}^{\prime} t t^{-1} \bar{W}_{j 2} t W_{j 2}^{-1} W^{\prime-1} W_{11}^{-1} t^{-1} .
$$

$\Theta_{1}$ is glued to $\Theta_{2}$ along the path with the label $\bar{V}, \Theta_{3}$ is glued to $\Theta_{2}$ along the path with the label $\bar{W}^{\prime}$. The union of $\Theta_{1}, \Theta_{2}$ and $\Theta_{3}$ has the same boundary label as $\Delta_{1}$.

Now instead of one maximal $\mathcal{G}$-subdiagram $\Theta$ we obtained three: $\Theta_{4}, \Theta_{5}$ and $\Theta_{6}$. where $\Theta_{4}$ has the boundary label $X_{1} \ldots X_{j-1} V, \Theta_{5}$ has the boundary label $V^{-1} W_{j 1} W^{\prime-1} W_{12}$ and $\Theta_{6}$ has the boundary label $W_{11} W^{\prime} W_{j 2} X_{j} \ldots X_{k}$. The diagram $\Theta_{5}$ is the interior of a $t$-annulus, and, together with this $t$-annulus, gives $\Theta_{2}$. We can end Transformation 5 by application of Transformation 1 and replacing $\Theta_{2}$ by a diagram over $\mathcal{U}$. This decreases the number of $t$-cells in $\Delta$, because instead of $\left|W_{12}\right|_{\mathcal{V}}+\left|W_{j 1}\right|_{\mathcal{V}}$ t-cells we have now $|V|_{\mathcal{V}}+\left|W^{\prime}\right|_{\mathcal{V}}$. This contradicts the assumption that $\Delta$ is minimal.

The case where the first possibility in Definition 3 holds can be considered similarly.

Suppose now that the third possibility holds. Then there is a $j \leq k$ such that $W_{1} \equiv W_{11} W_{12}$, with $W_{11} \neq 1$ and $W_{12} \neq 1$ both belonging to $\mathcal{V}, W_{j} \equiv W_{j 1} W_{j 2}$, with $W_{j 1} \neq 1$ and $W_{j 2} \neq 1$ both belonging to $\mathcal{V}$, and

$$
W_{12} X_{1} W_{2} X_{2} \ldots W_{j 1}=T
$$

where $T$ is not necessarily in $\mathcal{V}$, but $|T|<H$ and $W_{12}, W_{j 1}$ have minimal length among the subwords with this property (see Fig. 15 on p. 000). Then we can represent $\Theta$ as a union of two $\mathcal{G}$-subdiagrams $\Theta_{1}$ and $\Theta_{2}$, where $\Theta_{1}$ has contour label

$$
W_{12} X_{1} W_{2} X_{2} \ldots W_{j 1} T^{-1}
$$

and $\Theta_{2}$ has contour label

$$
W_{11} T W_{j 2} \ldots X_{k}
$$

If $W_{j 2}=W_{j 21} W_{j 22}, W_{11}=W_{111} W_{112}$ and $W_{j 22} \ldots X_{k} W_{111}=T_{1}$, where $T_{1} \notin \mathcal{V}$ but $\left|T_{1}\right|<H$, then $\Theta$ is the union of $\Theta_{1}$ and a $\mathcal{G}$-subdiagram $\Theta_{3}$ with contour 
(a)

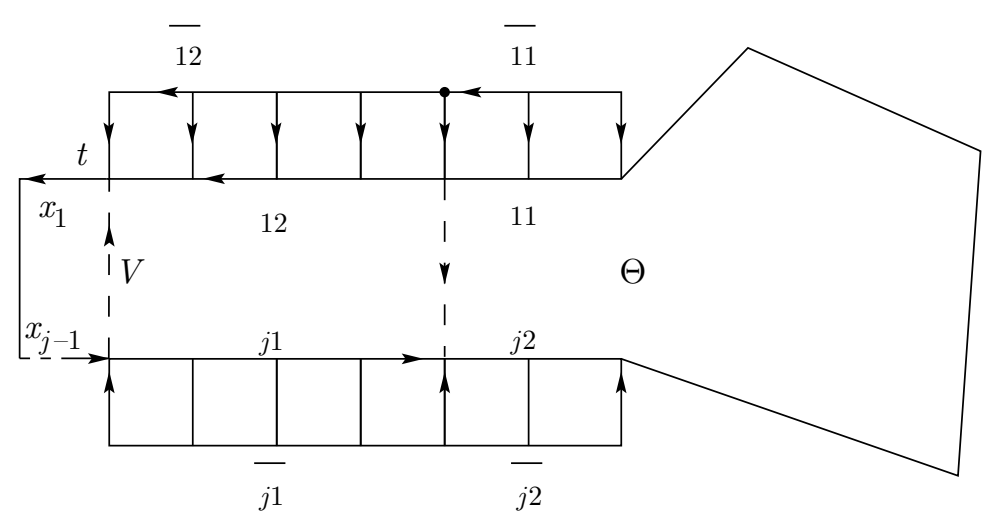

(b)
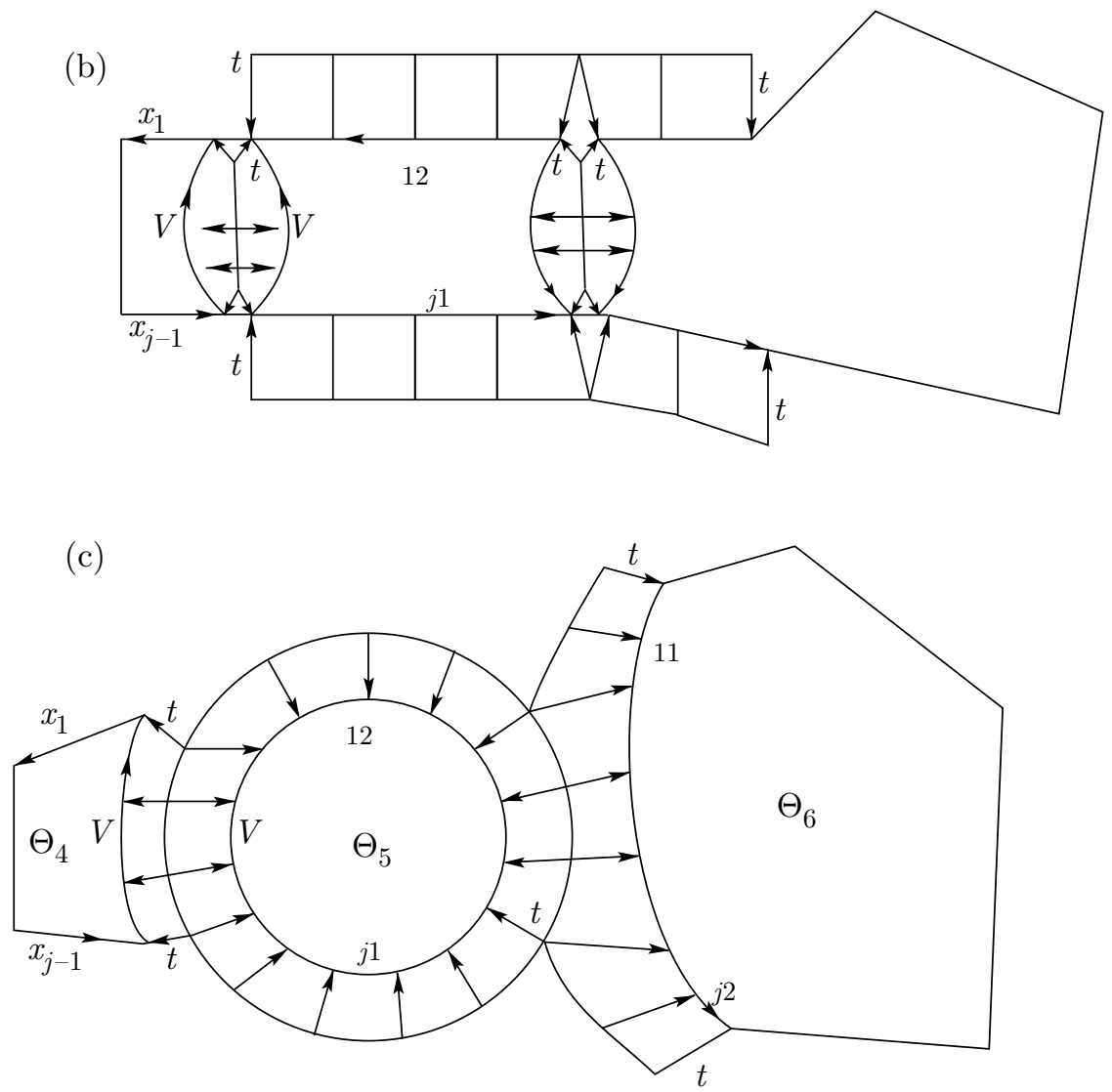

Figure 14

$W_{111} T_{1} W_{j 22} X_{j} \ldots X_{k}$, connected to $\Theta_{1}$ by a $\mathcal{V}$-piece with contour $T W_{j 21} T_{1}^{-1} W_{112}$, as shown on Fig. 15. 


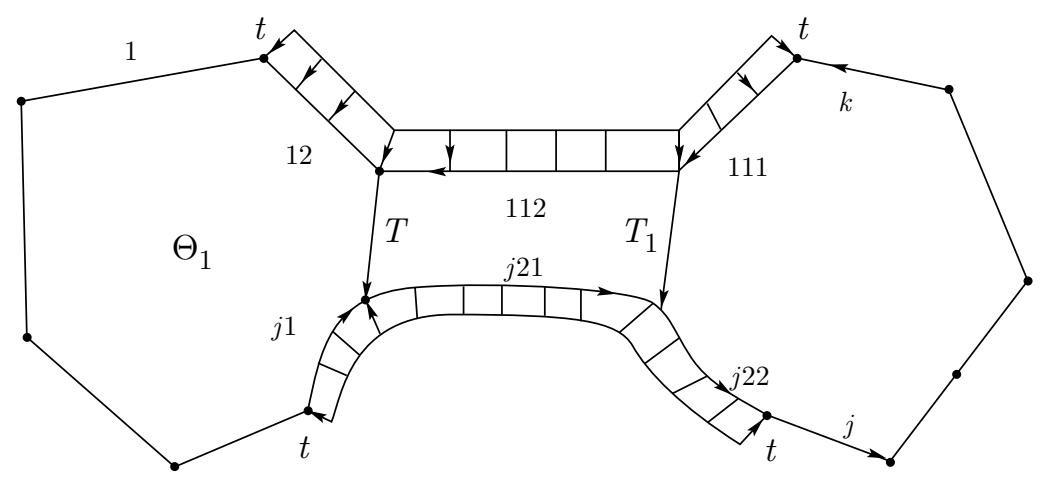

FiguRe 15

Every time when a diagram under consideration is splittable we represent it as a union of several subdiagrams. Continuing this process, we will obtain the desired partition.

Definition 9. A piece with a contour that does not contain any boundary paths is called a concealed piece.

Definition 10. A piece is called a $k$-piece if it has $k$ strippaths on the contour.

Lemma 10. Suppose we have fixed some partition of maximal $\mathcal{G}$-subdiagrams in $\Delta$ into nonsplittable pieces and $\mathcal{V}$-pieces. Let $N_{k}(k>2)$ be the number of nonsplittable $k$-pieces and $N_{2}$ be the number of nonsplittable 2-pieces plus the number of $\mathcal{V}$-pieces in $\Delta$, and let $S$ be the number of $t$-strips in it, $S \geq 3$. Then $J:=\sum_{k=2}^{S} N_{k} k \leq$ $15(S-2)$.

Proof. The number of concealed pieces between two $t$-strips cannot be more than one.

We say that two $t$-strips adjoin irregularly if they do not adjoin through phase vertices. A subdiagram with a contour formed by two $t$-strips adjoined irregularly is an island, but it is always either properly included in some nonsplittable piece or included in some $\mathcal{V}$-piece.

From now on, we will not worry about filling in the pieces by subdiagrams over $\mathcal{G}$; we just consider all possible configurations of $S t$-strips in the plane. We can forget that the strips are $t$-strips and think about them simply as strips. We treat the paths shorter than $H$ on the boundaries of $\mathcal{V}$-pieces as if they were just points, so that at these points the corresponding $t$-strips are tangent. The number $J$ will remain the same after this assumption.

We use induction on $S$. The cases $S=3,4$ we verify directly. (See Fig. 16 for the maximal possible values of $J$ for $S=3$,4.) Suppose that the lemma has been proved for diagrams with fewer than $S$ strips. Suppose that a diagram $\Theta$ has $S$ strips. Either there is a strip that splits the diagram into two parts $\Theta_{1}$ and $\Theta_{2}$ (see Fig. 17) with at least two strips in $\Theta_{1}$ and two strips in $\Theta_{2}$, or there is no such strip. If there is no such strip we just draw one more strip such that it splits the diagram into two parts $\Theta_{1}$ and $\Theta_{2}$ (see Fig. 17) and each of these parts contains at least two strips. Suppose that $\Theta_{1}$ contains $S_{1}$ strips and $\Theta_{2}$ contains $S_{2}$ strips; in the first case $S=S_{1}+S_{2}+1$, in the second case $S=S_{1}+S_{2}$. 

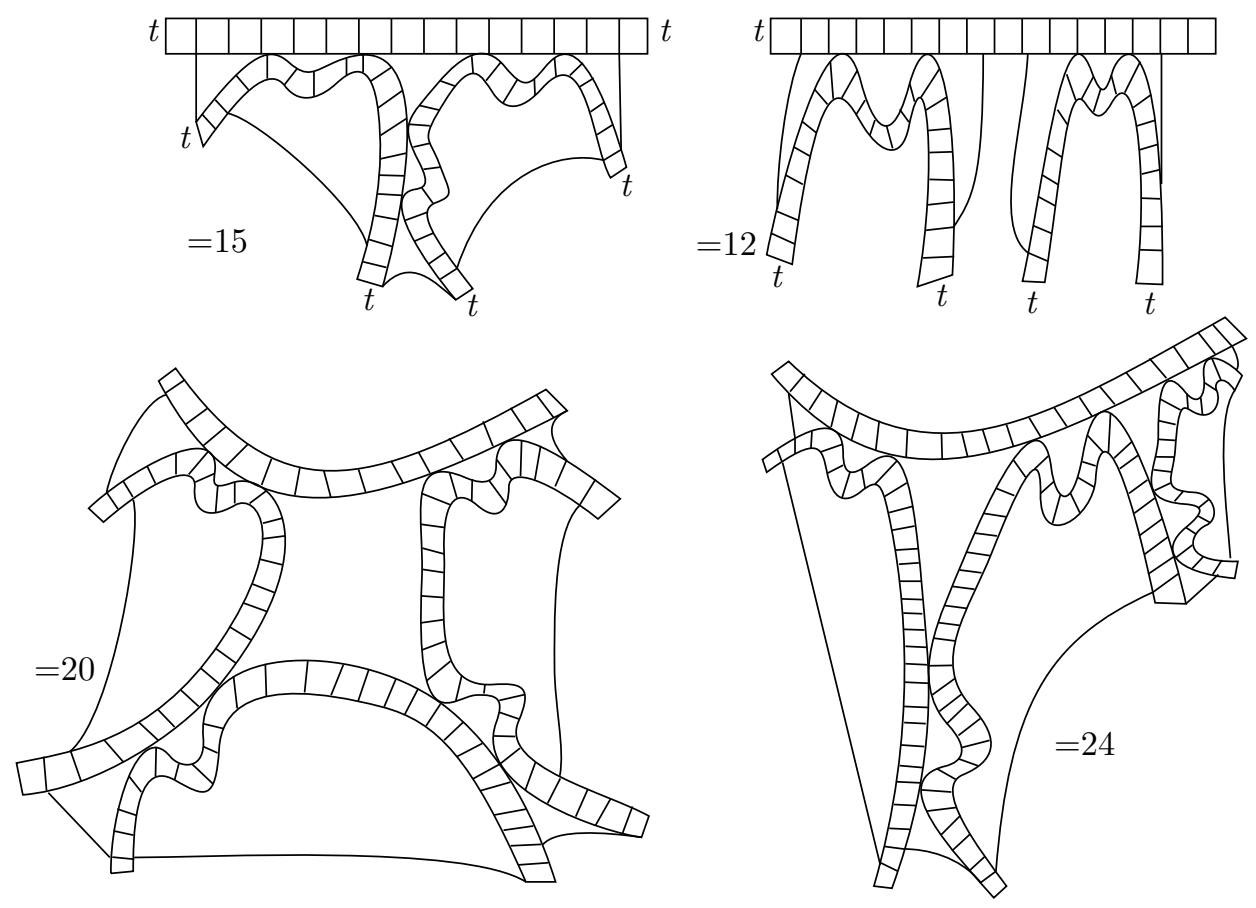

FiguRe 16
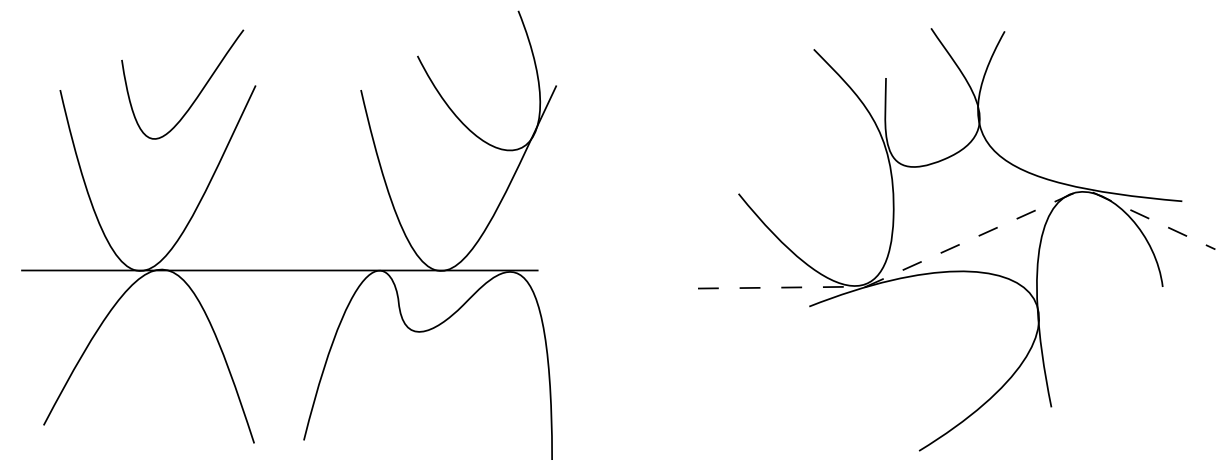

FiguRE 17

Apply the induction hypothesis to the union of $\Theta_{1}$ and the dividing strip. We have $J_{1}=\sum_{k=2}^{S_{1}+1} N_{k} k \leq 15\left(S_{1}+1-2\right)$. If we apply induction to the union of $\Theta_{2}$ and the fixed strip, then we have $J_{2}=\sum_{k=2}^{S_{2}+1} N_{k} k \leq 15\left(S_{2}+1-2\right)$. Upon summation, $J=\sum_{k=2}^{S} N_{k} k \leq J_{1}+J_{2} \leq 15\left(S_{1}+S_{2}-2\right) \leq 15(S-2)$.

Our goal now is to assign to each $t$-strip a set of nonsplittable pieces and $\mathcal{V}$-pieces in such a way that every piece occurs at most once in the union of these sets. 


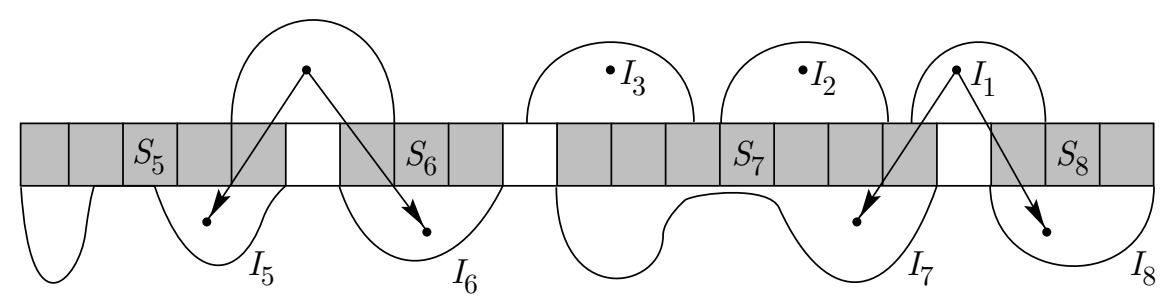

FigurE 18

Dual Forest. We construct a dual forest of $\Delta$ in the following way. We plot a vertex of the dual forest in each piece (recall that "piece" means a nonsplittable piece or a $\mathcal{V}$-piece).

Before defining the dual forest of $\Delta$, we consider the family $\mathcal{F}$ of all subdiagrams of $\Delta$ with the following property: if $\Theta \in \mathcal{F}$ contains some cell of a $t$-strip, then it contains the whole $t$-strip, and if $\Theta$ contains a cell of a maximal $G$-subdiagram, then it contains the whole subdiagram. We shall define the edges of the forest for subdiagrams in $\mathcal{F}$ by induction on the number of $t$-strips.

Definition 11. The piece part of a t-strip, corresponding to a piece $I$, is the maximal connected set of cells $\tau(I)$ in the $t$-strip that border the piece $I$ (i.e. having some path in common with the contour of the piece).

Definition 12. Two pieces $I$ and $J$ are called neighbours if $\tau(I) \cap \tau(J) \neq \emptyset$; in other words, if there is a cell in a $t$-strip such that its $\mathcal{U}$-side belongs to one piece and its $\mathcal{V}$-side to the other.

Suppose the subdiagram $\Theta$ has one $t$-strip (see Fig. 18).

We follow one of the sides of the $t$-strip (the $\mathcal{U}$-side, for instance) starting at one of the $t$-edges. As we meet the first piece $I_{1}$ (see Fig. 18), we draw directed edges from $I_{1}$ to all the neighbouring pieces, if there are any (in Fig. 18 these pieces are $I_{8}$ and $I_{7}$ ), and to each edge we associate the piece part of the $t$-strip determined by the piece representing the endpoint of the edge. We also color these associated piece parts.

$S_{8}$ is assigned to the edge $\left(I_{1}, I_{8}\right), S_{7}$ is assigned to the edge $\left(I_{1}, I_{7}\right)$. (On Fig. 18 we color $S_{7}$ and $S_{8}$.) Then we take the next piece $I$ along the $\mathcal{U}$-side of the $t$-strip. There are three possibilities: 1) $I$ does not have neighbouring pieces; 2) the piece part $\tau(I)$ is already coloured; 3$)$ the piece part $\tau(I)$ is not completely coloured and $I$ has neighbouring pieces.

In the first two cases we go to the next piece; in the third case we draw directed edges to the vertices in the neighbouring pieces. And again to each edge we associate the piece part of the $t$-strip determined by the piece representing the endpoint of the edge. We also color these associated piece parts.

We continue this process until we have exhausted all the pieces on the $\mathcal{U}$-side of the $t$-strip. Finally all the piece parts of the $t$-strip, associated to the pieces representing the endpoints of the edges, are colored. Every uncoloured cell of the $t$-strip has at least one side on the boundary of $\Theta$.

Now suppose that for subdiagrams from $\mathcal{F}$ that contain not more than $s$ strips we have an algorithm to construct the dual forest and simultaneously colour the piece parts of $t$-strips assigned to the edges of the dual forest. Assume furthermore that the algorithm is such that the following conditions are satisfied: 


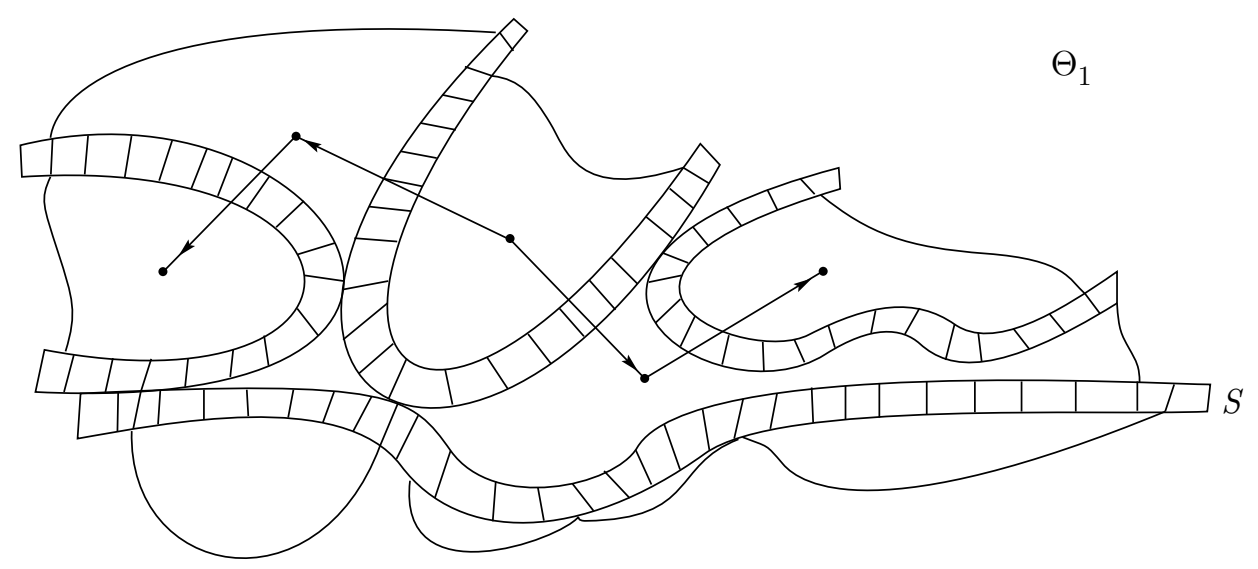

Figure 19

1. The piece parts of the $t$-strip, associated to the pieces representing the endpoints of the edges, are colored. Every uncoloured cell of the $t$-strip has at least one side on the boundary of the subdiagram.

2. A vertex of the forest cannot be an endpoint of two edges.

Now suppose we have $s+1 t$-strips in the subdiagram $\Theta$. Fix one $t$-strip $S$, such that there are no $t$-strips on one side of it. By a simple induction argument such a $t$-strip always exists. Suppose, for definiteness, that there are no $t$-strips on the $\mathcal{U}$-side of it. Consider the subdiagram $\Theta_{1}$ on the other side $(\mathcal{V}$-side) of this $t$-strip. This diagram contains $s$-strips (see Fig. 19) We suppose that the dual forest and the coloring for $\Theta_{1}$ have already been constructed and satisfy the induction hypothesis. Our purpose is to extend the dual forest to $\Theta$ and to colour the piece parts of the $t$-strip $S$.

Consider now the subdiagram $\Theta_{2}$ from $\mathcal{F}$ consisting of $S$ and the neighbouring maximal $G$-subdiagrams. We go from right to left along the $\mathcal{V}$-side of $S$, and repeat the procedure of drawing edges and colouring the piece parts of $S$ as in the first step of the induction.

The constructed graph is still a forest, because the graph in $\Theta_{1}$ is a forest by our induction assumption, and our construction is such that the new arrows do not produce cycles. Moreover, all the piece parts of $t$-strips in $\Theta$ are assigned to edges of the dual forest, the uncoloured parts have one side on the boundary of $\Theta$, and a vertex of the forest cannot be an endpoint of two edges.

Lemma 11. Suppose there is a nonsplittable $k$-piece in $\Delta$ with the contour label

$$
W \equiv W_{1} X_{1} W_{2} X_{2} \ldots W_{k} X_{k} .
$$

Let $X_{i_{1}}, \ldots, X_{i_{p}}$ be the labels of the parts of the boundary of this piece that are on the boundary of $\Delta$. Let $n_{i}$ be the number of $t$-cells corresponding to the word $W_{i}$. Let $M_{1}, M_{2}$ be the constants from Corollary 6. Then for any $M_{3} \geq M_{2}+2 c, M_{3} \geq H$, and for any $i$,

$$
\left|n_{i}\right| \leq M_{1} \sum_{j}\left|X_{i_{j}}\right|+M_{3} k
$$


Proof. This follows from Corollary 6 and the fact that for a nonsplittable piece the $X_{i_{j}}$ 's that do not belong to the boundary of $\Delta$ are rather short, shorter then $2 c$, hence their sum is less then $2 c k$.

Lemma 12. Let $E_{1}$ be the sum of all cells in all t-strips of $\Delta, D$ the length of the boundary of $\Delta$, and $S$ the number of $t$-strips (hence $D_{1}=D-2 S$ is the length of the part of the boundary excluding the t-edges). Let $M_{4}=(1+c \lambda)\left(15 M_{3}+M_{1}\right)$. Then $E_{1} \leq M_{4} D$.

Proof. Every $t$-strip is partitioned into piece parts and parts intersecting the boundary of $\Delta$. To each piece part we assign some nonsplittable piece or some maximal $\mathcal{V}$ - piece, namely the endpoint of the corresponding edge in the dual forest. To the remaining parts we assign the intersection with the boundary of $\Delta$.

The label of each part of a $t$-strip that is assigned to a $\mathcal{V}$-piece is shorter in $\mathcal{G}$ than $2 H+R$, where $R$ is the length of the other part of the $t$-strip on the boundary of this $\mathcal{V}$-piece, and this part of the $t$-strip is estimated already not in the $\mathcal{V}$-piece but in nonsplittable pieces (two $\mathcal{V}$-pieces cannot be neighbours). Recall that $H \leq M_{3}$.

So, from Lemma 11 we have that

$$
E_{1} \leq(1+c \lambda) M_{1} D_{1}+(1+c \lambda) M_{3} \sum_{k=2}^{S} N_{k} k .
$$
$M_{4} D$

By Lemma $10, J=\sum_{k=2}^{S} N_{k} k \leq 15(S-2)$. So $E_{1} \leq(1+c \lambda)\left(M_{1} D_{1}+15 M_{3}\right)=$

We now apply

Transformation 6. Replace each maximal $\mathcal{G}$-subdiagram $\Theta$ in $\Delta$ by a diagram with the same contour, but where the number of cells contained in it is not more than $K$ times the length of the contour of $\Theta$ (it is possible to do this, because $\mathcal{G}$ satisfies a linear isoperimetric inequality with the constant $K$ ).

Lemma 13. Let $E$ be the number of cells in $\Delta$, and $M_{5}=K\left(1+2 M_{4} c\right)+M_{4}$. Then

$$
E \leq M_{5} D
$$

Proof. If $E_{2}$ is the total number of cells in all maximal $\mathcal{G}$-subdiagrams, then

$$
E_{2} \leq K\left(D_{1}+2 M_{4} c D\right) \text {. }
$$

Finally

$$
E=E_{1}+E_{2} \leq M_{4} D+K\left(D_{1}+2 M_{4} c D\right)=M_{5} D
$$

The proposition and Theorem 1 are proved.

Proof of Corollary 1. Let $\mathcal{G}$ be a hyperbolic group, $\mathcal{A}$ and $\mathcal{B}$ isomorphic virtually cyclic subgroups, hence ([8], [14]) quasiisometrically embedded. Then the separated HNN-extension $\mathcal{H}=\left\langle\mathcal{G}, t \mid \mathcal{A}^{t}=\mathcal{B}\right\rangle$ is hyperbolic by Theorem 1 .

Now suppose that the HNN-extension $\mathcal{H}$ is not separated.

First, suppose that there is an element $g \in \mathcal{G}$ such that $\mathcal{A}^{g} \cap \mathcal{B}$ is infinite. Then there is an element $b=a^{g}$ of infinite order, where $b \in \mathcal{B}$ and $a \in \mathcal{A}$. Then, for some nonzero integers $m$ and $n$, we have $t^{-1} a^{n} t=b^{m}$; hence $t^{-1} a^{n} t=g^{-1} a^{m} g$ and

$$
\left(t g^{-1}\right)^{-1} a^{n} t g^{-1}=a^{m} \text {. }
$$

It follows from [8] (Corollary 8.2.c) that if, in a hyperbolic group, an element $y$ has infinite order, and $m$ and $n$ are nonzero integers, then the equation $x^{-1} y^{n} x=y^{m}$ 
implies that the subgroup generated by $x$ and $y$ is virtually cyclic. In particular, $|m|=|n|$ and we say that $x$ nearly commutes with $y^{m}$.

But in our case both elements $t g^{-1}$ and $a$ have infinite order and are not powers of the same element (because the reduced form of $\left(\mathrm{tg}^{-1}\right)^{k_{1}}$ in the HNN-extension $\mathcal{H}$ is different from the reduced form $a^{k_{2}}$ for any $k_{1}, k_{2}$ ). Hence the subgroup generated by $t g^{-1}$ and $a$ cannot be virtually cyclic.

Suppose now that neither $\mathcal{A}$ nor $\mathcal{B}$ is conjugate separated. Then, for some $g_{1} \in \mathcal{G} \backslash \mathcal{A}$ and $g_{2} \in \mathcal{G} \backslash \mathcal{B}$, both sets

$$
S_{1}=\left\{a \in \mathcal{A} \mid g_{1}^{-1} a g_{1} \in \mathcal{A}\right\}
$$

and

$$
S_{2}=\left\{b \in \mathcal{B} \mid g_{2}^{-1} b g_{2} \in \mathcal{B}\right\}
$$

are infinite. Since $S_{1}$ and $S_{2}$ are then infinite subgroups of the virtually cyclic groups $\mathcal{A}$ and $\mathcal{B}$, there are elements $c \in S_{1}$ and $d \in S_{2}$ of infinite order. The inclusion $g_{1}^{-1} c g_{1} \in \mathcal{A}$ implies that $g_{1}$ nearly commute with a power of $c$. Also, $g_{2}^{-1} d g_{2} \in \mathcal{B}$ implies that $g_{2}$ nearly commutes with a power of $d$. If $\mathcal{H}$ were hyperbolic we would be able to find numbers $m$ and $n$ such that $g_{1}^{-2} c^{m} g_{1}^{2}=c^{m}, g_{2}^{-2} d^{n} g_{2}^{2}=d^{n}$ and $t^{-1} c^{m} t=d^{n}$. It is easy to see that the subgroup $\left\langle\left(t g_{2}^{2} t^{-1} g_{1}^{2}\right)^{2}, c^{m}\right\rangle$ is free abelian, a contradiction.

\section{Free products with amalgamation}

The proof of Theorem 2 is quite similar and not as complicated as the proof of Theorem 1, so this section will be quite brief, more in the vein of a guided exercise than a proof.

Let $\mathcal{G}_{1}, \mathcal{G}_{2}$ and $\mathcal{U}, \mathcal{V}$ be as in Theorem $2, \mathcal{R}=\mathcal{G}_{1} * \mathcal{U}=\mathcal{V} \mathcal{G}_{2}$.

From the hyperbolicity of $\mathcal{G}_{1}$ and $\mathcal{G}_{2}$ it follows that there is a constant $K$ such that for any element $W_{1}=1\left(\right.$ resp. $\left.W_{2}=1\right)$ in $\mathcal{G}_{1}$ (resp. $\left.\mathcal{G}_{2}\right)$ there is a reduced diagram over $\mathcal{G}_{1}$ (resp. $\mathcal{G}_{2}$ ) with boundary label $W_{1}$ (resp. $W_{2}$ ) and number of cells $\leq K\left\|W_{1}\right\|\left(\leq K\left\|W_{2}\right\|\right)$.

Let $A=\left\{a_{1}, \ldots, a_{n}\right\}$ and $B=\left\{b_{1}, \ldots, b_{n}\right\}$ be the distinguished generating sets for $\mathcal{G}_{1}$ and $\mathcal{G}_{2}$ respectively, such that $\phi\left(a_{i}\right)=b_{i}$. The contents of this section is the proof of the following fact.

Proposition 3. There is a linear function $L_{1}$ of a single variable, depending only on $\mathcal{G}_{1}, \mathcal{G}_{2}, A, B$, such that for any element $W=1$ in $\mathcal{R}$ there is a diagram over $\mathcal{R}$ with boundary label $W$ and number of cells $\leq L_{1}(|| W||)$.

A $(\mathcal{U}, \mathcal{V})$-cell is a cell with contour $a_{i} b_{i}^{-1}$. In this section $(\mathcal{U}, \mathcal{V})$-cells will play the role of $t$-cells.

Let $\Delta$ be a minimal diagram over $\mathcal{R}$ with boundary label $W$ (this means that $\Delta$ contains the least possible number of $(\mathcal{U}, \mathcal{V})$-cells).

A $(\mathcal{U}, \mathcal{V})$-strip is a subdiagram, with boundary $a_{i_{1}} \ldots a_{i_{k}} b_{i_{k}}^{-1} \ldots b_{i_{1}}^{-1}$ consisting of $(\mathcal{U}, \mathcal{V})$-cells, that begins and ends on the boundary of the diagram $\Delta$, and is minimal with this property. $(\mathcal{U}, \mathcal{V})$-strips will play the role of $t$-strips. A $(\mathcal{U}, \mathcal{V})$-cell and $(\mathcal{U}, \mathcal{V})$-strip are shown in Fig. 20.

Lemma 14. A diagram $\Delta$ cannot contain a $(\mathcal{U}, \mathcal{V})$-annulus.

The proof follows from the minimality of $\Delta$.

The diagram $\Delta$ consists of maximal $\mathcal{G}_{1}$ - and $\mathcal{G}_{2}$-subdiagrams that are glued to each other through $(\mathcal{U}, \mathcal{V})$-strips. A typical form of $\Delta$ is shown in Fig. 21; the 


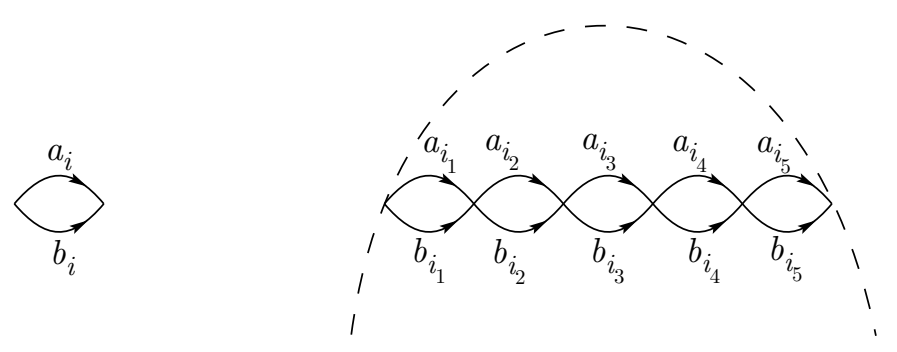

FIGURE 20

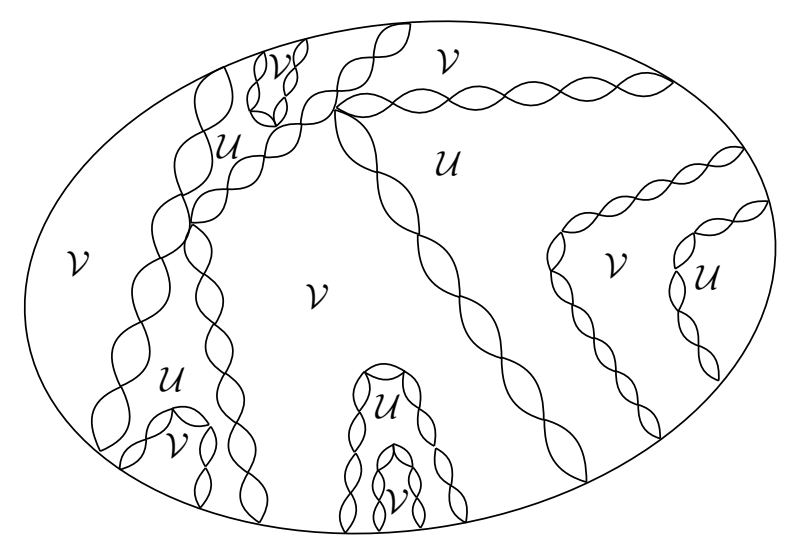

Figure 21

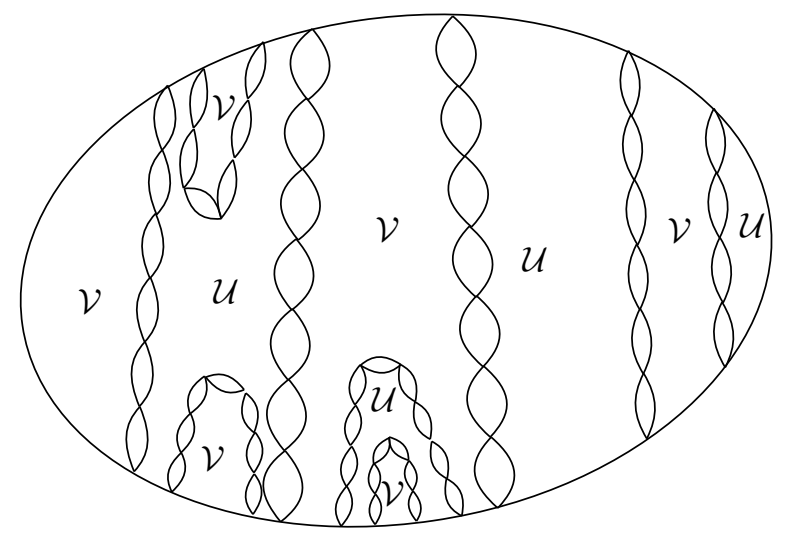

Figure 22

same diagram is schematically shown in Fig. 22 (the notion of a $(\mathcal{U}, \mathcal{V})$-strip is clear from Fig. 22). The partition of the set of all $(\mathcal{U}, \mathcal{V})$-cells into $(\mathcal{U}, \mathcal{V})$-strips is not necessary unique; we just take some partition.

The notions of an island, a nonsplittable piece (in a maximal $\mathcal{G}_{1}$ - or $\mathcal{G}_{2}$-subdiagram) and a $\mathcal{V}$-piece are the same as in the previous section. 
We perform Transformations 2 and 3 on $\Delta$. After each transformation we reduce $\Delta$ to a minimal diagram.

Lemma 15. Every maximal $\mathcal{G}_{1}$-subdiagram can be transformed into a disjoint union of nonsplittable pieces with boundary labels of the form

$$
W_{1} X_{1} W_{2} X_{2} \ldots W_{k} X_{k}=1 \text {, }
$$

where all the $X_{i}$ correspond to boundary paths.

The proof is a simpler version of the proof of Lemma 9, so we omit it.

We construct a dual forest as we did in the previous section, but instead of $\mathcal{V}$-pieces and nonsplittable pieces in $\mathcal{G}_{2}$-subdiagrams we just use maximal $\mathcal{G}_{2}$-subdiagrams.

Lemma 16. Suppose that $\Delta$ contains a nonsplittable $\mathcal{G}_{1}$-piece with the contour label

$$
W_{1} X_{1} W_{2} X_{2} \ldots W_{k} X_{k} \text {. }
$$

Let $n_{i}$ be the number of $(\mathcal{U}, \mathcal{V})$-cells corresponding to $W_{i}$. Then for $M_{6}=M_{1}+M_{2}$ (these are the constants from Corollary 6) and for any $i$,

$$
\left|n_{i}\right| \leq M_{6} \sum_{i}\left|X_{i}\right|
$$

Proof. All the $X_{i}$ 's are nonempty words (recall that if $X_{i}=1$, then we consider the union of two strippaths $W_{i}$ and $W_{i+1}$ as one strippath). Hence $\sum_{i}\left|X_{i}\right| \geq k$.

Lemma 17. Let $E_{1}$ be the sum of all the cells in all the $(\mathcal{U}, \mathcal{V})$-strips of $\Delta, M_{7}=$ $M_{6}+c \lambda M_{6}$, and let $D$ be the length of the boundary of $\Delta$. Then $E_{1} \leq M_{7} D$.

Proof. Every $(\mathcal{U}, \mathcal{V})$-strip is subdivided into parts such that each part is assigned either to some nonsplittable $\mathcal{G}_{1}$-piece, or to a path on the boundary of $\Delta$ not included in the boundary of some nonsplittable $\mathcal{G}_{1}$-piece, or to a maximal $\mathcal{G}_{2}$-subdiagram. Each nonsplittable $\mathcal{G}_{1}$-piece and each such path on the boundary of $\Delta$ cannot be assigned to more then one $(\mathcal{U}, \mathcal{V})$-strip, nor to more then one distinct part of this strip. The length of each piece of a $(\mathcal{U}, \mathcal{V})$-strip, assigned a maximal $\mathcal{G}_{2}$-subdiagram $\Theta$, is not greater in $\mathcal{G}$ than the sum of the lengths of all other parts of $(\mathcal{U}, \mathcal{V})$-strips in $\Theta$ (that are assigned to some nonsplittable $\mathcal{G}_{1}$-pieces or to parts of the boundary of $\Delta$ ) plus the length of all the boundary-paths of $\Theta$.

Suppose $M_{6} \geq 1$. From Lemma 16 we have that

$$
E_{1} \leq M_{6} D+c \lambda M_{6} D=M_{7} D .
$$

Now we apply to $\Delta$ the analog of Transformation 6 . We replace all maximal $\mathcal{G}_{i^{-}}$ subdiagrams by diagrams with the same contour, but where the number of cells is less then the length of the contour times $K$.

Lemma 18. Let $E$ be the number of cells in $\Delta$, and $M_{8}=K\left(1+2 M_{7} c\right)+M_{7}$. Then

$$
E \leq M_{8} D
$$

Proof. If $E_{2}$ is the sum of the cells in all maximal $\mathcal{G}_{i \text {-subdiagrams, then }}$

$$
E_{2} \leq K\left(1+2 M_{7} c\right) D \text {. }
$$

Finally,

$$
E=E_{1}+E_{2} \leq M_{7} D+K\left(1+2 M_{7} c D\right)=M_{8} B .
$$

The proposition and Theorem 2 now follow from Lemma 18. 


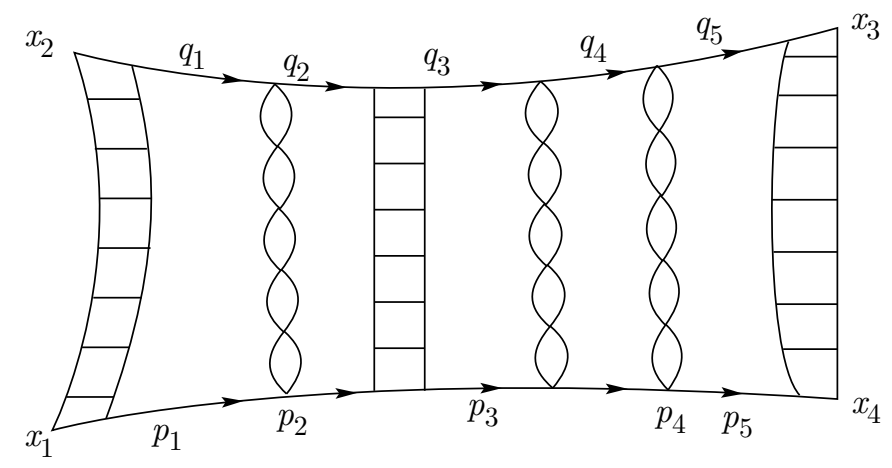

FiguRE 23

Proof of Corollary 2. In one direction, Corollary 2 follows from Theorem 2 and the fact that virtually cyclic subgroups of hyperbolic groups are quasiisometrically embedded.

Suppose now that $\mathcal{A}$ is not conjugate separated in $\mathcal{G}_{1}$ and $\mathcal{B}$ is not conjugate separated in $\mathcal{G}_{2}$. Then for some $g_{1} \in \mathcal{G}_{1} \backslash \mathcal{A}$ and $g_{2} \in \mathcal{G}_{2} \backslash \mathcal{B}$ both sets

$$
S_{1}=\left\{a \in \mathcal{A} \mid g_{1}^{-1} a g_{1} \in \mathcal{A}\right\}
$$

and

$$
S_{2}=\left\{b \in \mathcal{B} \mid g_{2}^{-1} b g_{2} \in \mathcal{B}\right\}
$$

are infinite. Since $S_{1}$ and $S_{2}$ are then infinite subgroups of the virtually cyclic groups $\mathcal{A}$ and $\mathcal{B}$, there are elements of infinite order $c \in S_{1}$ and $d \in S_{2}$. The inclusion $g_{1}^{-1} c g_{1} \in \mathcal{A}$ implies that $g_{1}$ nearly commutes with a power of $c$. Also, $g_{2}^{-1} d g_{2} \in \mathcal{B}$ implies that $g_{2}$ nearly commutes with a power of $d$. There is a common power $z$ of $c$ and $d$ such that $g_{1}$ and $g_{2}$ both nearly commute with $z$. It is easy to see that the subgroup $\left\langle\left(g_{1} g_{2}\right)^{2}, z\right\rangle$ is free abelian, a contradiction.

\section{Other SUFFICIENT CONDITIONS}

Let $\mathcal{H}$ be the fundamental group of a finite graph $\Gamma$ of groups (relative to some maximal subtree $T$ of $\Gamma$ ) with vertex groups $\mathcal{G}_{i}, i=1, \ldots p$, edge groups $\mathcal{U}_{i j}$ such that $\mathcal{U}_{i j} \leq \mathcal{G}_{i}$, and embeddings $\tau: \mathcal{U}_{i j} \rightarrow \mathcal{G}_{j}$ such that $\tau\left(\mathcal{U}_{i j}\right)=\mathcal{V}_{i j}=\mathcal{U}_{j i} \leq \mathcal{G}_{j}$. Then $\mathcal{H}$ is generated by the groups $\mathcal{G}_{i}$ and additional elements $t_{i j}$, which are in bijective correspondence with the non- $T$ edges. $\mathcal{H}$ has, in addition to the relations of groups $\mathcal{G}_{i}$, the following defining relations: $u=\tau(u)$ for any $u \in \mathcal{U}_{i j}$, with $(i j)$ a $T$-edge, and $u^{t_{i j}}=\tau(u)$ for all $u \in \mathcal{U}_{i j}$ with $(i j)$ a non $T$-edge.

Definition 13. A diagram of the type shown in Fig. 23 is called an $h$-rectangular subdiagram over $\mathcal{H}$, if the following conditions are satisfied:

1. The strips are either $\left(\mathcal{U}_{i j}, \mathcal{V}_{i j}\right.$ )-strips or $t_{i j}$-strips (which we will also call $\left(\mathcal{U}_{i j}, \mathcal{V}_{i j}\right)$-strips).

2. The subdiagrams between strips are maximal $\mathcal{G}_{i}$-subdiagrams.

3. The boundary-paths $p_{k}, q_{k}$ are shorter than some fixed number $h$.

4. If the labels of the strippaths of a maximal $\mathcal{G}_{i}$-subdiagram belong to the same edge group $\mathcal{U}_{i j}$, and both strips that bound it are $\left(\mathcal{U}_{i j}, \mathcal{V}_{i j}\right)$-strips, then the label of at least one of its two boundary paths does not belong to $\mathcal{U}_{i j}$. 
The number $n$ of $\left(\mathcal{U}_{i j}, \mathcal{V}_{i j}\right)$-strips in the diagram is called the length of the diagram; the paths $\left[x_{1}, x_{2}\right],\left[x_{3}, x_{4}\right]$ are called the sides of the diagram. The lengths of the labels of the sides are taken in the corresponding edge groups. If the labels of the two boundary paths of each maximal $\mathcal{G}_{i}$-subdiagram are the same $\left(\phi\left(p_{k}\right)=\phi\left(q_{k}\right)\right)$, then the diagram is called a conjugacy h-rectangular diagram.

We obtain sufficient conditions for the hyperbolicity of $\mathcal{H}$ which are weaker than the conditions in Theorems 1 and 2.

Theorem 3. Let $\mathcal{H}$ be the fundamental group of a finite graph of groups, with the edge groups $\mathcal{U}_{i j}$ quasiisometrically embedded in the corresponding vertex groups $\mathcal{G}_{i}$ and $\mathcal{G}_{j}$ (E-quasiconvex). Suppose that all the vertex groups $\mathcal{G}_{i}$ are hyperbolic, and $\delta$ is the maximum of the hyperbolicity constants of the vertex groups. Let $H=8 \delta+\varepsilon$. If there exists a number $n$ such that only a finite number of elements in $\mathcal{H}$ can be labels of the sides of a reduced conjugacy $2 H$-rectangular diagram of length $n$, then $\mathcal{H}$ is hyperbolic.

The condition of the theorem implies that all the elements in this finite set have finite order.

We will prove the theorem after formulating the following corollaries.

Corollary 7. Let $\mathcal{H}$ be a fundamental group of a finite graph of groups, with edge groups $\mathcal{U}_{i j}$ quasiisometrically embedded in the corresponding vertex groups $\mathcal{G}_{i}$ and $\mathcal{G}_{j}$ Suppose that all the vertex groups $\mathcal{G}_{i}$ are torsion-free hyperbolic, and $\delta$ is the largest of the constants of hyperbolicity of the vertex groups. Let $H=8 \delta+\varepsilon$. If there exists a number $n$ such that there are no reduced conjugacy $2 H$-rectangular diagrams of length $n$, then $\mathcal{H}$ is hyperbolic.

Corollary 8. Let $\mathcal{G}_{1}, \mathcal{G}_{2}$ be hyperbolic groups, $\mathcal{U} \leq \mathcal{G}_{1}, \mathcal{V} \leq \mathcal{G}_{2}$ quasiisometrically embedded, and $\phi: \mathcal{U} \rightarrow \mathcal{V}$ an isomorphism. Suppose that there exists a number $n$ such that the set

$$
h_{n} \ldots\left(g_{2}\left(\phi^{-1}\left(h_{1}\left(\phi\left(g_{1} \mathcal{U} g_{1}^{-1} \cap \mathcal{U}\right)\right) h_{1}^{-1} \cap \mathcal{V}\right)\right) g_{2}^{-1} \cap \mathcal{U}\right) \ldots h_{n}^{-1} \cap \mathcal{V}
$$

is finite (here all $g_{i} \in \mathcal{G}_{1} \backslash \mathcal{U}, h_{i} \in \mathcal{G}_{2} \backslash \mathcal{V}$ ). Then the group $\mathcal{G}_{1} * \mathcal{U}=\mathcal{V} \mathcal{G}_{2}$ is hyperbolic.

For HNN-extensions $\left\langle\mathcal{G}, t \mid \mathcal{U}^{t}=\phi(\mathcal{U})=\mathcal{V}\right\rangle$ there is a more complicated condition:

Corollary 9. Let $\mathcal{G}$ be a hyperbolic group, $\mathcal{U} \leq \mathcal{G}, \mathcal{V} \leq \mathcal{G}$ quasiisometrically embedded, and $\phi: \mathcal{U} \rightarrow \mathcal{V}$ an isomorphism. Suppose there exists a number $n$ such that for any $C_{1}, \ldots, C_{n} \in\{\mathcal{U}, \mathcal{V}\}$ the set

$$
\left(g_{n} \ldots\left(\phi^{\alpha_{3}}\left(g_{2}\left(\phi^{\alpha_{2}}\left(g_{1} C_{1} g_{1}^{-1} \cap C_{2}\right)\right) g_{2}^{-1} \cap C_{3}\right)\right) \ldots g_{n}^{-1}\right) \cap C_{n+1}
$$

is finite. Here, if $C_{i}=C_{i+1}$ then $g_{i} \notin C_{i} ; \alpha_{i}=1$ if $C_{i}=\mathcal{U}$ and $\alpha_{i}=-1$ if $C_{i}=\mathcal{V}$.

Then the group $\mathcal{H}=\left\langle\mathcal{G}, t \mid \mathcal{U}^{t}=\phi(\mathcal{U})=\mathcal{V}\right\rangle$ is hyperbolic.

Proof of the theorem. We will prove that any diagram over $\mathcal{H}$ satisfies a linear isoperimetric inequality. The idea behind the proof is exactly the same as the idea of the proof of Theorems 1 and 2 .

It can be shown, as in the proof of Lemma 4, that if the sides of any minimal conjugacy $2 H$-rectangular diagram of length $n$ are bounded by a constant $C$, then there is a number $\bar{C}$ such that the sides of any minimal $2 H$-rectangular diagram of length $n$ are bounded by $\bar{C}$. (If they are not bounded, then one can find infinitely 
many elements that are the labels of the sides of conjugacy $2 H$-rectangular diagrams.) Let $\Delta$ be a minimal diagram over $\mathcal{H}$. It is subdivided by $\left(\mathcal{U}_{\alpha \beta}, \mathcal{V}_{\alpha \beta}\right)$-strips into maximal $\mathcal{G}_{\alpha}$-subdiagrams. The contour of each $\mathcal{G}_{\alpha}$-subdiagram is a word

$$
W_{1} X_{1} W_{2} X_{2} \ldots W_{k} X_{k},
$$

where $W_{1}, \ldots, W_{k} \in\left\{\mathcal{U}_{\alpha \beta}, \mathcal{V}_{\gamma \alpha} \mid \alpha, \beta, \gamma \in\{1, \ldots, p\}\right\}$.

We shall give a slightly different definition of a nonsplittable decomposition, than in Section 3. First, the following:

Definition 14. Given a decomposition

$$
W \equiv W_{1} X_{1} W_{2} X_{2} \ldots W_{k} X_{k}
$$

where $W_{1}, \ldots, W_{k} \in\left\{\mathcal{U}_{\alpha \beta}, \mathcal{V}_{\gamma \alpha} \mid \alpha, \beta, \gamma=1, \ldots, p\right\}$, the $W_{i}$ are geodesic in the corresponding groups, the $X_{i} \in \mathcal{G}_{\alpha}$ are reduced, and if $W_{i}, W_{i+1} \in \mathcal{U}_{\alpha \beta}\left(\mathcal{V}_{\gamma \alpha}\right)$, then $X_{j} \neq 1$, we call the decomposition a $\mathcal{G}_{\alpha}$-edges decomposition.

If $W=1$, and the indices in the above definition are taken modulo $k$, then it is called a cyclic $\mathcal{G}_{\alpha}$-edges decomposition.

Definition 15. A cyclic $\mathcal{G}_{\alpha}$-edges decomposition is called splittable if one of the following holds:

1. There is a $j \leq k$ such that $W_{1} \equiv W_{11} W_{12}, W_{11}, W_{12} \in \mathcal{U}_{\alpha \beta}, W_{j} \equiv W_{j 1} W_{j 2}$, $W_{j 1}, W_{j 2} \in \mathcal{U}_{\alpha \beta}$, and

$$
W_{12} X_{1} W_{2} X_{2} \ldots W_{j 1}=W^{\prime} \in \mathcal{U}_{\alpha \beta},
$$

where

$$
\left|W_{12}\right|_{\mathcal{U}_{\alpha \beta}}+\left|W_{j 1}\right|_{\mathcal{U}_{\alpha \beta}}>\left|W^{\prime}\right|_{\mathcal{U}_{\alpha \beta}}+\left|W_{j 1} W^{\prime-1} W_{12}\right|_{\mathcal{U}_{\alpha \beta}} .
$$

2. There is a $j \leq k$ such that $W_{1} \equiv W_{11} W_{12}, W_{11}, W_{12} \in \mathcal{V}_{\gamma \alpha}, w_{j} \equiv w_{j 1} w_{j 2}$, $w_{j 1}, w_{j 2} \in \mathcal{V}_{\gamma \alpha}$, and

$$
W_{12} X_{1} W_{2} X_{2} \ldots W_{j 1}=W^{\prime} \in \mathcal{V}_{\gamma \alpha},
$$

where

$$
\left|W_{12}\right|_{\mathcal{V}_{\gamma \alpha}}+\left|W_{j 1}\right|_{\mathcal{V}_{\gamma \alpha}}>\left|W^{\prime}\right|_{\mathcal{V}_{\gamma \alpha}}+\left|W_{j 1} W^{\prime-1} W_{12}\right|_{\mathcal{V}_{\gamma \alpha}} .
$$

3. There is a $j \leq k$ such that $W_{1} \equiv W_{11} W_{12} W_{13}, W_{11} \neq 1, W_{13} \neq 1, W_{11}, W_{12}$, $W_{13} \in \mathcal{U}_{\alpha \beta}\left(\mathcal{V}_{\gamma \alpha}\right), W_{j} \equiv W_{j 1} W_{j 2} W_{j 3}, W_{j 1} \neq 1, W_{j 3} \neq 1 W_{j 1}, W_{j 2}, W_{j 3} \in$ $\mathcal{U}_{\alpha \beta_{1}}\left(\mathcal{V}_{\gamma_{1} \alpha}\right)$ and

$$
\begin{gathered}
W_{13} X_{1} W_{2} X_{2} \ldots W_{j 1}=T, \\
W_{j 3} X_{j} \ldots W_{11}=T_{1},
\end{gathered}
$$

where $|T|,\left|T_{1}\right|<H$. In this case we always take pairs $W_{11}, W_{j 3}$ and $W_{13}, W_{j 1}$ of minimal length (in the corresponding edge groups) among the pairs with the same property. (The length of the pair $W_{k}, W_{t}$ is not less than the length of the pair $\bar{W}_{k}, \bar{W}_{t}$ if the length of $W_{k}$ is not less than the length of $\bar{W}_{k}$ and the length of $W_{t}$ is not less than the length of $\bar{W}_{t}$.)

A cyclic decomposition of $W$ is called cyclically nonsplittable if all the permutations of the form

$$
W_{i} X_{i} \ldots W_{k} X_{k} W_{k+1} \ldots W_{i-1} X_{i-1}=1 .
$$

are nonsplittable. 
As in Section 3, a maximal nonsplittable $\mathcal{G}_{i}$-subdiagram is called a nonsplittable piece.

Definition 16. A thin bridge is a $\mathcal{G}_{i}$-subdiagram having boundary label

$$
W_{1} X_{1} W_{2} X_{2}
$$

where the $X_{i}$ 's are shorter than $H$ (the $X_{i}$ 's can be trivial) and the $W_{i}$ 's are labels of strip-paths and geodesic in the corresponding edge groups; moreover, the thin bridge is required to be maximal among such $\mathcal{G}_{i}$-subdiagrams.

Lemma 19. Every maximal G-subdiagram in $\Delta$ consists of nonsplittable pieces connected by thin bridges.

The proof is very similar to the proof of Lemma 9. The diagram $\Delta$ is minimal hence cannot contain a $\left(\mathcal{U}_{i j}, \mathcal{V}_{i j}\right)$-annulus. One can perform on $\Delta$ the obvious analogs of Transformations 2 and 3.

We can now construct a dual forest in $\Delta$ as we did for HNN-extensions, but instead of nonsplittable pieces and $\mathcal{V}$-pieces we use nonsplittable pieces and thin bridges.

Lemma 20. There are constants $M_{1}, M_{2}$ such that if, in a vertex group $\mathcal{G}_{\alpha}$, we have a cyclically nonsplittable decomposition of the word

$$
W \equiv W_{1} X_{1} W_{2} X_{2} \ldots W_{k} X_{k}=1,
$$

then, for $W_{i} \in \mathcal{U}_{\alpha \beta}\left(\mathcal{V}_{\gamma \alpha}\right)$, we have $\left|W_{i}\right|_{\mathcal{U}_{\alpha \beta}\left(\mathcal{V}_{\gamma \alpha}\right)} \leq M_{1} \sum_{i}\left|X_{i}\right|+M_{2} k$.

The proof is similar to the proof of Corollary 6 .

The proof of the following lemma repeats the proof of Lemma 10.

Lemma 21. Let $N_{k}$ be the number of nonsplittable $k$-pieces if $k \geq 3$, and let $N_{2}$ be the number of nonsplittable 2-pieces plus the number of thin bridges in a diagram $\Delta$ over the group $\mathcal{H}$. Let $S$ be the number of $\left(\mathcal{U}_{i j}, \mathcal{V}_{i j}\right)$-strips in it, $S \geq 3$. Then $J=\sum_{k=2}^{S} N_{k} k \leq 15(S-2)$.

Notice that $S$ is no larger than $p$ (the number of vertex groups) times the sum of the number of distinct boundary-paths of $\Delta$ and the number of $t_{i j}$-edges on the boundary of $\Delta$; hence, if $D$ is the length of the boundary of $\Delta$, then $S \leq p D$.

The proof of the following lemma repeats the proof of Lemma 11.

Lemma 22. Suppose we have a nonsplittable $k$-piece in $\Delta$ with the contour label

$$
W \equiv W_{1} X_{1} W_{2} X_{2} \ldots W_{k} X_{k} .
$$

Let $X_{i_{1}}, \ldots, X_{i_{p}}$ be pieces of the boundary of $\Delta$. Let $n_{i}$ be the number of $\left(\mathcal{U}_{\alpha \beta}, \mathcal{V}_{\alpha \beta}\right)$ cells corresponding to the word $W_{i}$. Then, for any $M_{3} \geq M_{2}+2 c$ and for any $i$, we have

$$
\left|n_{i}\right| \leq M_{1} \sum_{j}\left|X_{i_{j}}\right|+M_{3} k
$$

In constructing the dual forest we assigned to each piece part of each $\left(\mathcal{U}_{\alpha \beta}, \mathcal{V}_{\alpha \beta}\right)$ strip an edge of the dual forest. This edge is associated either with some nonsplittable piece or with some thin bridge containing the endpoint of the edge.

The lengths of those parts of the $\left(\mathcal{U}_{\alpha \beta}, \mathcal{V}_{\alpha \beta}\right)$-strips that are assigned to the edges having endpoints in nonsplittable pieces, are estimated in these pieces. The only 


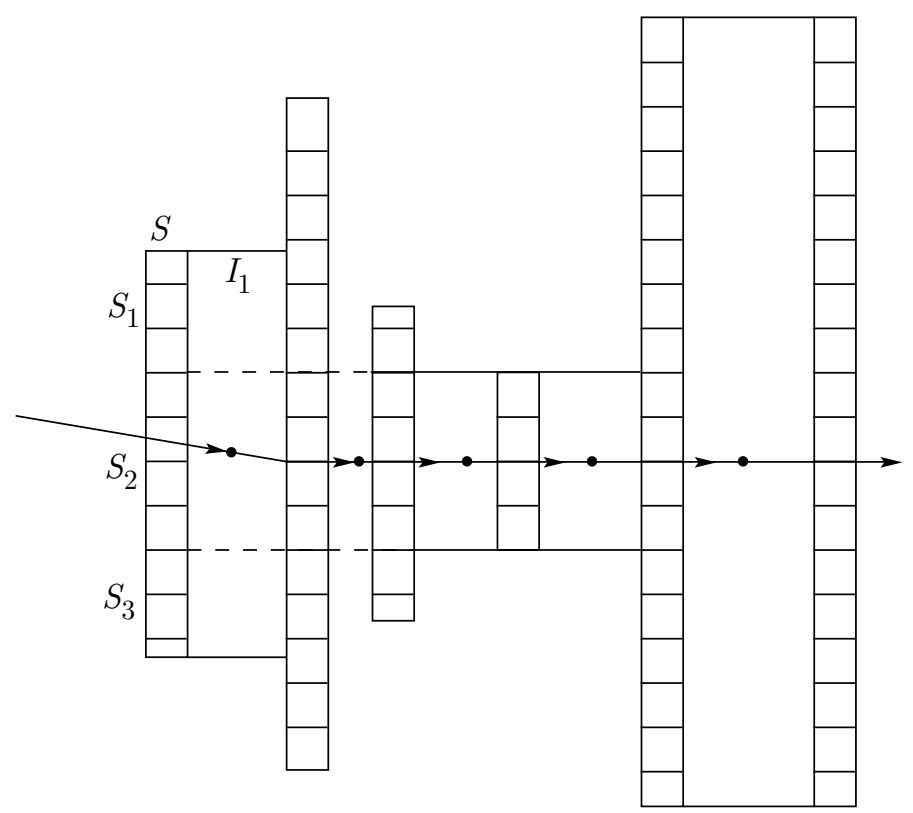

FiguRe 24

problem is to estimate the length of the $\left(\mathcal{U}_{\alpha \beta}, \mathcal{V}_{\alpha \beta}\right)$-strips assigned to edges having endpoints in thin bridges.

Consider the configuration of thin bridges as shown in Fig. 24. The arrows correspond to the edges of the dual forest.

The sides of any $2 H$-rectangular diagram of length $n$ are bounded by $\bar{C}$. Take the part of the $\left(\mathcal{U}_{\alpha \beta}, \mathcal{V}_{\alpha \beta}\right)$-strip $S$ assigned to the piece associated with the endpoint of the first edge $\left(I_{1}\right)$. $S$ can be split into parts, such that for each part one of the following possibilities applies:

1. we can construct a $2 H$-rectangular subdiagram of length more than $n$ starting with this part;

2. the length of the rectangular subdiagram connecting this part of the strip $S$ with some part of a strip $R$, assigned to an edge of the dual forest with endpoint in some nonsplittable piece, is less than $n$ (then the length of this part can be bounded by the length of $R$ times some constant $C_{1}$, depending only on $H$ and $n$ ).

Now, taking $M_{3}>\bar{C}$ and $M_{4}=n C_{1} M_{1}+15 n p M_{3}$, we have that the number of all $\left(\mathcal{U}_{\alpha \beta}, \mathcal{V}_{\alpha \beta}\right)$-cells in $\Delta$ is less than $M_{4} D$, where $D$ is the length of the boundary of $\Delta$. And, taking $M_{5}$ as in Lemma 13, we get the linear isoperimetric inequality for $\Delta$ with the constant $M_{5}$. The theorem has been proved.

To prove the corollaries it suffices to notice that the conditions in the statements of the corollaries imply the conditions of the theorem. 


\section{Some RESUlts on QUASICONVEXITY}

A subset $Y$ in a geodesic space $\Gamma$ is called quasiconvex for some $\epsilon \geq 0$ if every geodesic segment $\left[y_{1}, y_{2}\right]$ with endpoints in $Y$ lies $\epsilon$-close to $Y$. A subgroup $U$ of a group $G$ is called quasiconvex if $\Gamma(U)$ is quasiconvex in $\Gamma(G)$.

In this section we prove the following theorems.

Theorem 4. Let $\mathcal{H}=\left\langle\mathcal{G}, t \mid \mathcal{U}^{t}=\mathcal{V}\right\rangle$ be hyperbolic with $\mathcal{U}$ quasiconvex in $\mathcal{H}$. Then $\mathcal{G}$ is quasiconvex in $\mathcal{H}$, and hence hyperbolic.

Theorem 5. Let $\mathcal{H}$ be a separated $H N N$-extension, $\mathcal{H}=\left\langle\mathcal{G}, t \mid \mathcal{U}^{t}=\mathcal{V}\right\rangle$, with $\mathcal{G}$ hyperbolic, $\mathcal{U}$ and $\mathcal{V}$ quasiconvex in $\mathcal{G}$. Then $\mathcal{G}$ is quasiconvex in $\mathcal{H}$.

Proof of Theorem 4. We have to show that there exists a number $\lambda$ such that the length of arbitrary geodesic in $\mathcal{G}$ is shorter than $\lambda$ times the length of the same element in $\mathcal{H}$. Let $W$ be a geodesic word in $\mathcal{G}$ and $V$ a geodesic word in $\mathcal{H}$ such that $V=W$ in $\mathcal{H}$. Let $p$ be a path such that $\phi(p)=W$, and $q$ a path such that $\phi(q)=V$. The subgroup $\mathcal{U}$ is quasiconvex in $\mathcal{H}$, hence $\mathcal{V}$ is quasiconvex in $H$. Let $\lambda$ be a number such that every geodesic in $\mathcal{U}$ or $\mathcal{V}$ is $\lambda$-quasigeodesic in $\mathcal{H}$. Let $\Delta$ be a minimal diagram over $\mathcal{H}$ with the boundary $q p^{-1}$. Then a typical form of $\Delta$ is shown in Fig. 25. The path $p$ is shorter than the path $s$ in Fig. 25, but the path $s$ is shorter than $\lambda|q|$.

The theorem is proved.

Exactly the same reasoning can be used to prove the following more general result.

Suppose we have a finite graph of groups, with finitely generated edge groups, and the fundamental group $\mathcal{H}$ of the graph is hyperbolic. It then follows that if for some vertex group $\mathcal{G}(v)$ all incoming edge groups are quasiconvex in the whole group $\mathcal{H}$, then the vertex group $\mathcal{G}(v)$ is quasiconvex in $\mathcal{H}$. The result in this formulation was obtained by I. Kapovich (who used a different technique) [9].

Proof of Theorem 5. We will show that if $L$ is a linear function as in Proposition 2 , then the length of an arbitrary geodesic in $\mathcal{G}$ is shorter than $L(\ell)$, where $\ell$ is the length of the same element in $\mathcal{H}$. Let $W$ be a geodesic word in $\mathcal{G}$ and $V$ a geodesic word in $\mathcal{H}$ such that $V=W$ in $\mathcal{H}$. Let $p$ be a path such that $\phi(p)=W$ and $q$ a path such that $\phi(q)=V$. Let $\Delta$ be a minimal diagram over $\mathcal{H}$ with the boundary $q p^{-1}$. Then a typical form of $\Delta$ is shown in Fig. 25. We construct the dual forest for $\Delta$ starting from the pieces between $p$ and $s$ as shown in Fig. 26. Then $s$ is shorter than $L(|q|)$ and $p$ is shorter than $s$.

The following theorems can be proved by a similar technique.

Theorem 6. Let $\mathcal{H}=\mathcal{G}_{1} *_{\mathcal{U}} \mathcal{G}_{2}$ be a hyperbolic group, with $\mathcal{U}$ quasiconvex in $\mathcal{H}$. Then $\mathcal{G}_{1}$ and $\mathcal{G}_{2}$ are quasiconvex in $\mathcal{H}$, and hence hyperbolic.

Theorem 7. Let $\mathcal{H}=\mathcal{G}_{1} * \mathcal{U}=\mathcal{V} \mathcal{G}_{2}$, with $\mathcal{G}_{1}$ and $\mathcal{G}_{2}$ hyperbolic, $\mathcal{U}$ quasiconvex in $\mathcal{G}_{1}, \mathcal{V}$ quasiconvex in $\mathcal{G}_{2}$ and $\mathcal{U}$ conjugate separated in $\mathcal{G}_{1}$. Then $\mathcal{G}_{1}$ and $\mathcal{G}_{2}$ are quasiconvex in $\mathcal{H}$.

In the situation where $\mathcal{U}$ is malnormal in $\mathcal{G}_{1}$ and $\mathcal{V}$ is malnormal in $\mathcal{G}_{2}$, this result can also be deduced from [15]. 


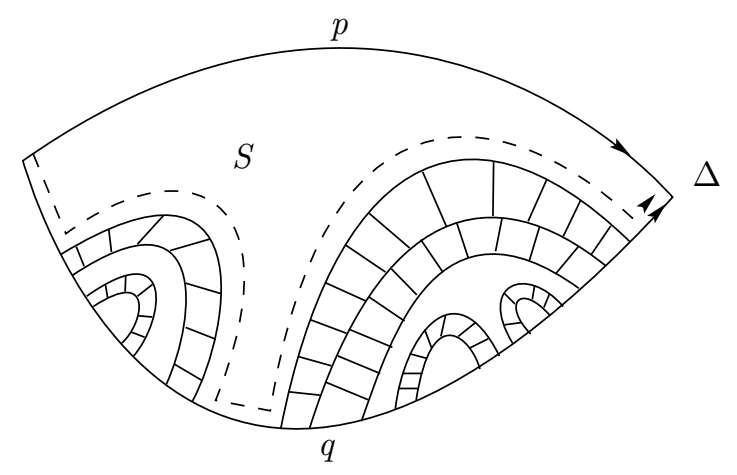

FIGURE 25

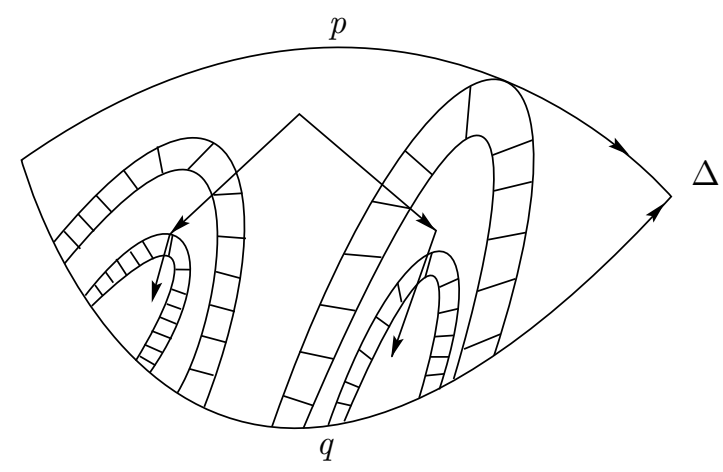

Figure 26

\section{Applications to exponential gRoups}

Let $A$ be an arbitrary associative ring with identity and $G$ a group. Fix an action of the ring $A$ on $G$, i.e. a map $G \times A \rightarrow G$. The result of the action of $\alpha \in A$ on $g \in G$ is written as $g^{\alpha}$. Consider the following axioms:

1. $g^{1}=g, g^{0}=1,1^{\alpha}=1$;

2. $g^{\alpha+\beta}=g^{\alpha} \cdot g^{\beta}, g^{\alpha \beta}=\left(g^{\alpha}\right)^{\beta}$;

3. $\left(h^{-1} g h\right)^{\alpha}=h^{-1} g^{\alpha} h$;

4. $[g, h]=1 \Longrightarrow(g h)^{\alpha}=g^{\alpha} h^{\alpha}$.

Definition 17. Groups with $A$-actions satisfying axioms 1)-4) are called $A$-groups.

In particular, an arbitrary group $G$ is a $\mathbf{Z}$-group. We now recall the definition of $A$-completion in the case where $A$ is the field of rationals $\mathbf{Q}$. Such completions were studied by G. Baumslag in [2], [1]. A-completions for arbitrary rings $A$ were investigated in [12]. We will use some results and constructions from the latter article.

Definition 18. Let $G$ be a group . Then a $\mathbf{Q}-$ group $G^{\mathbf{Q}}$ together with a homomorphism $G \rightarrow G^{\mathbf{Q}}$ is called a tensor $\mathbf{Q}$-completion of the group $G$ if $G^{\mathbf{Q}}$ satisfies the following universal property: for any $\mathbf{Q}-$ group $H$ and a homomorphism $\varphi: G \rightarrow H$ there exists a unique $\mathbf{Q}$-homomorphism $\psi: G^{\mathbf{Q}} \rightarrow H$ (a homomorphism that commutes with the action of $\mathbf{Q}$ ) such that the following diagram commutes: 


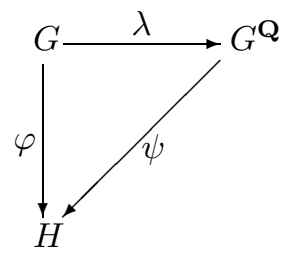

It was proved in [2] (see also [12]) that for every group $G$ the tensor $\mathbf{Q}$-completion of $G$ exists and is unique.

In this section we describe the $\mathbf{Q}$-completion $G^{\mathbf{Q}}$ of a torsion-free hyperbolic group $G$ as the union of an effective chain of hyperbolic subgroups. This allows one to apply techniques of hyperbolic group theory to solve various algorithmic problems in $G^{\mathbf{Q}}$, in particular, to construct effectively some natural normal forms for its elements (induced by the normal forms of amalgamated free products).

First of all, let us describe the construction of the complete tensor extension of centralizers of an arbitrary torsion-free hyperbolic group $G$ by the ring $\mathbf{Q}$ (see [12]).

Let $C=C_{G}(v)=C(v)$ be a centralizer in $G$ and $v$ not a proper power, i.e. $C(v)=\langle v\rangle$. The $\mathbf{Q}$-extension of the centralizer $C$ is by definition a free product with amalgamation

$$
G(C, \mathbf{Q})=G *_{C} \mathbf{Q},
$$

where $C \simeq \mathbf{Z} \leq \mathbf{Q}$. The group $G(C, \mathbf{Q})$ can be obtained as a union of a chain of subgroups,

$$
G=G_{0}(v)<G_{1}(v)<\ldots<G_{n}(v) \ldots,
$$

where $G_{i+1}(v)=G_{i}(v) *_{v_{i}=v_{i+1}^{i+1}}\left\langle v_{i+1}\right\rangle$; here $v_{0}=v$. In other words, $G(C, \mathbf{Q})$ can be obtained from $G$ as a union of a countable sequence of elementary extensions of centralizers of the type

$$
E(H, v, m)=H *_{v=w^{m}}\langle w\rangle,
$$

where the subgroup $\langle v\rangle$ is maximal abelian in $H$. If $X$ is a fixed set of generators of $H$, then we will consider the set $X \cup\{w\}$ as a canonical set of generators for $E(H, v, n)$. The length function on $E(H, v, n)$, introduced below, is associated with this set of generators.

A cyclically minimal element $v$ of a group $G$ is called a primitive element if it is not a proper power.

For an arbitrary group $G$ and a natural number $n \geq 2$ choose a set of elements $\mathcal{V}_{n}=\left\{v_{1} \ldots v_{t}\right\}$ satisfying the following condition $\left(S_{n}\right)$ :

1) $\mathcal{V}_{n}$ consists of primitive elements of length not more than $n$;

2) no two centralizers in the set of centralizers $\mathcal{C}_{n}=\left\{C(v) \mid v \in \mathcal{V}_{n}\right\}$ are conjugate in $G$;

3) the set $\mathcal{V}_{n}$ is maximal with respect to properties 1) and 2); i.e., any element of length not more than $n$ is conjugate to a power of some $v \in \mathcal{V}_{n}$.

By definition, the group $G\left(\mathcal{C}_{n}\right)$ is the union of the finite chain of groups

$$
G<E\left(G, v_{1}, n\right)=G_{1}<E\left(G_{1}, v_{2}, n\right)=G_{2}<\ldots<E\left(G_{t-1}, v_{t}, n\right)=G_{t}=G\left(\mathcal{C}_{n}\right) ;
$$

thus $G\left(\mathcal{C}_{n}\right)$ is obtained from $G$ by consecutive extensions of centralizers from $\mathcal{C}_{n}$ :

$$
\left.\left.G\left(\mathcal{C}_{n}\right)=\left(\ldots\left(G *_{v_{1}=w_{1}^{n}}\left\langle w_{1}\right\rangle\right) *_{v_{2}=w_{2}^{n}}\left\langle w_{2}\right\rangle\right) * \ldots\right) *_{v_{t}=w_{t}^{n}}\left\langle w_{t}\right\rangle\right) .
$$

Notice that this definition does not depend on the order of the elements in $\mathcal{C}_{n}$. 
Lemma 23. Let $G\left(\mathcal{C}_{n}\right)$ be as above. Then there exists a set $\mathcal{V}_{n+1}$ in $G\left(\mathcal{C}_{n}\right)$ that satisfies the condition $\left(S_{n+1}\right)$ and contains $\left\{w_{1}, \ldots, w_{t}\right\}$.

Proof. The elements $w_{1}, \ldots, w_{t}$ are simple in $G\left(\mathcal{C}_{n}\right)$ because their length is equal to 1 . They are pairwise nonconjugate in $G\left(\mathcal{C}_{n}\right)$. Indeed, from the description of conjugate elements in free products with amalgamation $([10])$, one can derive the following assertion: Let $g$ be a cyclically reduced element in $E(H, v, n)$. Suppose $g$ is conjugate in $E(H, v, n)$ to some element in $H$. Then $g \in H$.

Applying this fact to the chain (2), one can verify the pairwise nonconjugacy of the $w_{i}$ 's. This proves the lemma.

We now intend to exhibit $G^{\mathbf{Q}}$ as a union of a chain of groups

$$
G=T_{0}<T_{1}<T_{2}<\ldots<\bigcup_{n=0}^{\infty} T_{n},
$$

defined by induction on $n$ as follows. Assume that the groups $T_{i}, i<n$, and the sets $\mathcal{V}_{i} \subset T_{i-1}$ have already been constructed and satisfy the condition $\left(S_{i}\right)$ in $T_{i-1}$. If $\mathcal{V}_{n}=\left\{v_{1}, \ldots, v_{t}\right\}$, then

$$
\left.\left.T_{n}=\left(\ldots\left(T_{n-1} * v_{1}=w_{1}^{n}\left\langle w_{1}\right\rangle\right) *_{v_{2}=w_{2}^{n}}\left\langle w_{2}\right\rangle\right) * \ldots\right) * v_{t}=w_{t}^{n}\left\langle w_{t}\right\rangle\right)
$$

i.e. $T_{n}=T_{n-1}\left(\mathcal{C}_{n}\right)$, where $\mathcal{C}_{n}$ is the corresponding set of centralizers. By the previous lemma there exists a subset $\mathcal{V}_{n+1} \subset T_{n}$ which satisfies the condition $\left(S_{n+1}\right)$ in $T_{n}$ and contains $w_{1}, \ldots, w_{t}$.

Definition 19. We will call a sequence of groups $H_{1}, H_{2}, \ldots$ effective if there is an algorithm wich allows one, for any $i$, to construct a finite representation of the group $H_{i}$.

Theorem 8. Let $G$ be a torsion-free hyperbolic group and

$$
G=T_{0}<T_{1}<T_{2}<\ldots
$$

the sequence of groups described above; then

1. $T_{n}$ is hyperbolic for any $n$,

2. $\left\{T_{n}\right\}_{n \in \mathbf{N}}$ is an effective sequence of groups,

3. $\bigcup_{n=0}^{\infty} T_{n}=G^{\mathbf{Q}}$.

Proof. 1. By our construction, $T_{n+1}$ can be obtained from $T_{n}$ by a finite sequence of extensions of centralizers of the type $E(H, v, m)=H *_{v=w^{m}}\langle w\rangle$, where the subgroup $\langle v\rangle$ is maximal abelian in $H$. Suppose $H$ is torsion-free hyperbolic; then $\langle v\rangle$ is conjugate separated in $H$ and, by Corollary $2, E(H, v, m)$ is also hyperbolic. $T_{0}=G$ and $G$ is hyperbolic; hence, by induction, all $T_{n}$ are hyperbolic. Moreover, the proof of Theorem 2 shows that the constant of hyperbolicity $\delta(E(H, v, m))$ can be effectively found if we know a finite presentation of $H$. This means that for any $n$ we can effectively find the constant of hyperbolicity of the group $T_{n}$, if we know such a constant for $G$.

2. Recall that $T_{n+1}=T_{n}\left(\mathcal{C}_{n+1}\right)$; so, having a finite presentation for $T_{n}$ and an effective procedure to construct the set of elements $\mathcal{V}_{n}$, we can effectively construct a finite presentation for $T_{n+1}$ (see the presentation (2) above).

Lemma 24. If $G$ is a torsion-free hyperbolic group, then there is an algorithm to construct the set $\mathcal{V}_{n}$ for any number $n$. 
Proof. To prove the assertion we need the solvability in any hyperbolic group of the word and conjugacy problems as well as the power problem; i.e. the problem of deciding if an element is a proper power in $G$. The word and conjugacy problems are solved in [8].

To solve the power problem we will use the quasigeodesic property of powers in hyperbolic groups; namely, there is a constant $\lambda>0$ such that $\left|W^{n}\right| \geq \lambda n|W|$ for any cyclically minimal word $W$ (see [14], Lemma 1.12, and [13], Lemma 27). Examining the proofs of these lemmas, one sees that $\lambda$ can be found effectively as a function of $\delta$ and $|A|$, where $A$ is the distinguished system of generators of $G$. In light of the solvability of the word and conjugacy problems, it is possible to decide if an element is cyclically minimal, and to enumerate all cyclically minimal elements in accordance with increasing length. Note that if a cyclically minimal element $V$ is equal to $W^{n}$, then $W$ is cyclically minimal and $n|W| \leq \lambda|V|$. Therefore, to determine if a cyclically minimal element $V$ is a proper power, we enumerate all cyclically minimal elements $W$ such that $|W| \leq \lambda|V|$, and for each $n$ such that $n|W| \leq \lambda|V|$ we verify the equality $W^{n}=V$. This shows that we can effectively list all simple elements of length not greater than $n$. Now, to create a set $\mathcal{V}_{n}$ one only needs to list all simple elements of length not greater than $n$, and delete those which are conjugate to previous ones. This can be done effectively because of the decidability of the conjugacy problem.

3. As we mentioned above, every element in $\mathcal{V}=\bigcup \mathcal{V}_{n}$ has arbitrary roots in the union $T=\bigcup_{n=0}^{\infty} T_{n}$. Moreover, every centralizer $C_{T}(v), v \in \mathcal{V}$, is isomorphic to the additive group of $\mathbf{Q}$, so it admits an action of $\mathbf{Q}$ satisfying the module axioms. By our construction, every centralizer in $T$ is conjugate to the centralizer of an element $v \in \mathcal{V}$. Hence we can, through conjugation, induce an action of $\mathbf{Q}$ on all centralizers in $T$; i.e. on the group $T$. This action is defined unambiguously, because different centralizers in $T$ have trivial intersection (see [12]). Hence $T$ is a $\mathbf{Q}$-group. Using the universal property of free products with amalgamation, one can prove that this group satisfies the universal property of a $\mathbf{Q}$-completion of $G$. See [12] for details. The theorem has been proved.

Let us now discuss algorithmic problems over groups of type $G^{\mathbf{Q}}$. Formally, $\mathbf{Q}$-groups can be considered as groups with operators from $\mathbf{Q}$. This means that the language of $\mathbf{Q}$-groups contains group multiplication and countably many operations $f_{\alpha}, \alpha \in \mathbf{Q}$ (here, by definition, $f_{\alpha}(g)=g^{\alpha}$ for any $g \in G$ ). There are free objects (free $\mathbf{Q}$-groups) in the variety of $\mathbf{Q}$-groups, so, as usual, one can consider presentations of $\mathbf{Q}$-groups in the variety of $\mathbf{Q}$-groups.

Lemma 25. Let $\langle X \mid R\rangle$ be a presentation of a group $G$. Then the group $G^{\mathbf{Q}}$ has the same presentation $\langle X \mid R\rangle$ in the variety of $\mathbf{Q}$-groups.

The proof follows from the fact that the $\mathbf{Q}$-group with presentation $\langle X \mid R\rangle$ and $G^{\mathbf{Q}}$ have the same universal property in the category of $\mathbf{Q}$-groups.

Therefore, Q-completions of hyperbolic groups have finite presentations as Qgroups. Let $A=\left\{a_{1}, \ldots, a_{m}\right\}$ be a generating set for $G$. Then the elements in $G^{\mathbf{Q}}$ can be represented in the language of $\mathbf{Q}$-groups by words in the alphabet $A$. We are now able to formulate naturally the word problem, the conjugacy problem, the equations problem and the isomorphism problem for finitely presented Q-groups. Furthermore, when we say that some algorithm is applied to some elements or groups, this means that the algorithm is applied to the corresponding $\mathbf{Q}$-words and $\mathbf{Q}$-presentations. We would like to reduce the algorithmic problems for $G^{\mathbf{Q}}$ to the 
hyperbolic groups $T_{n}$. To this end we need to construct an algorithm to determine for any element $g \in G^{\mathbf{Q}}$ the number $n(g)$ such that $g \in T_{n(g)}$. We will do this at the same time as we construct normal forms for elements from $G^{\mathbf{Q}}$. These normal forms derive from the construction of $G^{\mathbf{Q}}$, starting from $G$, as a countable iteration of extensions by free products with amalgamation. First of all, we introduce normal forms for the elements in the group

$$
E(H, v, m)=H *_{v=w^{m}}\langle w\rangle,
$$

which is the elementary extension of a centralizer $C(v)=\langle v\rangle$ in $H$ by the adjunction of an $m^{\text {th }}$ root to $v$. It will be convenient to denote $w$ by $v^{\frac{1}{m}}$. Let $S_{m}=\left\{\frac{k}{m} \mid k=\right.$ $1, \ldots, m-1\}$. With this notation, the set $\left\{v^{s} \mid s \in S_{m}\right\}$ is a system of representatives in $\langle w\rangle$ for the cosets of $\langle v\rangle$.

Definition 20. A sequence of elements $\left(h_{1}, v^{s_{1}}, h_{2}, \ldots, v^{s_{n}}, h_{n+1}\right)$, where $h_{i} \in$ $H, s_{i} \in S_{m}$, is a semicanonical form of an element $g \in E(H, v, m)$ if

$$
g=h_{1} v^{s_{1}} \cdots v^{s_{n}} h_{n+1}
$$

and $h_{i} \notin\langle v\rangle, i=2, \ldots, n$.

Lemma 26. Any two semicanonical forms of $g$ can be transformed into each other by a finite sequence of commutations of the form $v^{s} v^{t}=v^{t} v^{s}$, where $t \in \mathbf{Z}, s \in S_{m}$.

The proof follows from the definition of reduced forms for elements of a free product with amalgamation (see [12] for details).

Taking fixed right coset representatives of the subgroup $\langle v\rangle$ in $H$ as the elements $h_{i}, i=2, \ldots, n$, in (4) we obtain the notion of canonical form of $g$.

Suppose now that the notions of canonical and semicanonical forms of elements of the group $H$ have already been introduced. One can then extend them to the group $E(H, v, m)$.

We will say that the semicanonical (resp. canonical) forms of an element

$$
g=h_{1} v^{s_{1}} \cdots v^{s_{n}} h_{n+1}
$$

in $E(H, v, m)$ agree with those on $H$, iff the elements $h_{1}, \ldots, h_{n+1}, v$ are in semicanonical (resp. canonical) form in the group $H$. By definition an element $h \in H$ has the same semicanonical (resp. canonical) form in $E(H, v, m)$ as in $H$.

Now let us consider the union of a chain of groups

$$
G=G_{0}<G_{1}<\ldots<G_{n}<\ldots<\bigcup_{n=0}^{\infty} G_{n}
$$

where every $G_{n+1}$ is obtained from $G_{n}$ by an elementary extension of a centralizer (i.e. it is an extension of the type $\left.E\left(H, v_{n+1}, m_{n+1}\right)\right)$, and the notions of semicanonical and canonical forms on $G_{n+1}$ are compatible with those on $G_{n}$. The forms on the terms of the chain induce corresponding well-defined forms on the resulting group $\bigcup_{n=0}^{\infty} G_{n}$. Note that the described forms depend on the chosen elements $v_{n}, n \in \mathbf{N}$, and numbers $m_{n}, n \in \mathbf{N}$.

By our construction, the $\mathbf{Q}$-completion $G^{\mathbf{Q}}$ of a torsion-free hyperbolic group $G$ is the union of the chain

$$
G=T_{0}<T_{1}<\ldots<T_{n}<\ldots,
$$

where $T_{n+1}$ is obtained from $T_{n}$ by a finite sequence of elementary extensions of centralizers. Hence, to introduce the semicanonical and canonical forms on $G^{\mathbf{Q}}$ it is enough to introduce them on $G$. 
Definition 21. Let $A$ be the generating set of $G$. The canonical (as well as the semicanonical) form of an element $g \in G$ is an $A$-word of miminal length representing $g$. The corresponding forms on $G^{\mathbf{Q}}$ (as described above) are called induced semicanonical (canonical) forms. These forms depend on the chosen sets $\mathcal{V}_{n}, n \in \mathbf{N}$, of elements $v_{i}$.

Let us suppose that some fixed sets of elements $\mathcal{V}_{n}, n \in \mathbf{N}$, have been chosen.

Lemma 27. Every element $g \in G^{\mathbf{Q}}$ has a semicanonical form of the type

$$
\left(x_{1}, v_{1}^{s_{1}}, x_{2}, \ldots, v_{m}^{s_{m}}, x_{m}\right),
$$

where $x_{i} \in G, v_{i} \in \bigcup \mathcal{V}_{n}, s_{i} \in \bigcup S_{n}$.

The proof by induction is straightforward.

By Definition 20, a semicanonical form of an element $g \in G^{\mathbf{Q}}$ is a sequence of group elements $\left(h_{1}, v^{s_{1}}, h_{2}, \ldots, v^{s_{n}}, h_{n+1}\right)$. When discussing algorithmic problems in the variety of $\mathbf{Q}$-groups, we will also consider semicanonical forms as sequences of $\mathbf{Q}$-words representing the corresponding elements. Moreover, if the sets of words $\mathcal{V}_{n}, n \in \mathbf{N}$, are fixed, then the words representing elements $v_{i}$ in semicanonical form must be fixed words from $\mathcal{V}_{n}$, and not arbitrary words representing $v_{i}$ in $G^{\mathbf{Q}}$.

Lemma 28. There is an algorithm which for every element $g \in G^{\mathbf{Q}}$, given in semicanonical form, computes a number $n=n(g)$ such that $g \in T_{n}$.

Proof: According to Lemma 24, there exists an algorithm which, for each $n$, lists the elements of the set $\mathcal{V}_{n}$ (i.e. it lists $\mathbf{Q}$-words in the alphabet $A$ representing these elements). For a given semicanonical form $\left(x_{1}, v_{1}^{s_{1}}, x_{2}, \ldots, v_{m}^{s_{m}}, x_{m}\right)$ one can effectively find for each $v_{i}$ a number $n_{i}$ such that $v_{i} \in \mathcal{V}_{n_{i}}$. By our construction, this semicanonical form represents an element from the subgroup $T_{n}$, where $n$ is the maximal number in the set consisting of all $n_{i}$ 's and the denominators of all $s_{i}$ 's.

Theorem 9. Let $G$ be a torsion-free hyperbolic group. Then there are algorithms that, for any element $g \in G^{\mathbf{Q}}$, produce its semicanonical and canonical forms with respect to some fixed family of sets $\mathcal{V}_{n}, n \in \mathbf{N}$.

Proof. Let us fix an arbitrary family of sets $\mathcal{V}_{n}, n \in \mathbf{N}$ (which can be computed by some algorithm). For any $\mathbf{Q}$-word $W$ in the alphabet $A$, representing some element $g \in G^{\mathbf{Q}}$, one needs to construct effectively the canonical form of $g$. We will argue by induction on the depth of the word $W$. The depth of $W$ is a positive integer $d(W)$, defined by induction: $d(a)=1$ for any $a \in A ; d\left(W_{1} W_{2}\right)=\max \left\{d\left(W_{1}\right), d\left(W_{2}\right)\right\}$ for any $\mathbf{Q}$-words $W_{1}, W_{2} ; d\left(W^{\alpha}\right)=d(W)+1$, where $\alpha \in \mathbf{Q} \backslash \mathbf{Z}$. It is easy to see that there exists an algorithm which for any $\mathbf{Q}$-word $W$ calculates $d(W)$.

Let $d(W)=1$; then $W$ represents an element from the initial group $G$. In light of the decidability of the word problem in $G$, one can effectively construct a canonical form of $g$, i.e. a minimal word in the alphabet $A$ representing the element $g$.

Let $d(W)>1$. Then $W=W_{1}^{r_{1}} \ldots W_{h}^{r_{h}}$, where $d\left(W_{i}\right)<d(W), r_{i} \in \mathbf{Q}$, and the $\mathbf{Q}$-words $W_{1}, \ldots, W_{h}$ can be found effectively from $W$. By induction, we can effectively determine for any $i$ the semicanonical form of $W_{i}$ and therefore determine a number $n$ such that $W_{i} \in T_{n}$. By the definition of the sets $\mathcal{V}_{n}, n \in \mathbf{N}$, if an integer $k$ is greater then the length of all $W_{i}$ in the generators of the group $T_{n}$, then any $W_{i}$ is conjugate to some power of an element $v_{i}$ from the set $\mathcal{V}_{n+k}$. By Lemma 24 the elements from the set $\mathcal{V}_{n+k}$ can be listed effectively. So, looking through all words 
$x$ (for example, according to increasing lengths), one can find effectively the words $v_{i} \in \mathcal{V}_{n+k}$, some integers $l_{i}$, and words $x_{i}$ such that $W_{i}=x_{i}^{-1} V_{i}^{l_{i}} x_{i}$ in the group $T_{n+k}$. Using axiom 3) from the definition of an $A$-group, the word $W$ represents in $T_{n+k}$ the same element as the word

$$
x_{1}^{-1} V_{1}^{l_{1} r_{1}} x_{1} \ldots x_{k}^{-1} V_{k}^{l_{k} r_{k}} x_{k},
$$

and the latter word is constructed effectively from $W$. So it is left only to transform it into semicanonical form. The procedure for this is the following: find equal neighbours $V_{i}=V_{i+1}=V$ (if any) and verify if the word $x_{i} x_{i+1}^{-1}$ between them belongs to the cyclic subgroup $\langle V\rangle$ (This can be done effectively, as explaned before). If $x_{i} x_{i+1}^{-1}=V^{t}$ for some integer $t$, then replace the subword $V^{r_{i} l_{i}} x_{i} x_{i+1}^{-1} V^{r_{i+1} l_{i+1}}$ by the word $V^{q}$, where $q=r_{i} l_{i}+r_{i+1} l_{i+1}+t$. And if $q=m+\frac{s_{1}}{s_{2}}$, where $m$ is an integer and $0<s_{1}<s_{2}$, then replace $V^{q}$ by the word $V^{m} V^{s_{1} / s_{2}}$. The resulting word represents the same element as the original word, but the number of $V_{i}$ 's in it is less than in the original one. Arguing by induction, we complete the process. It is not hard to see that the resulting word will be in semicanonical form. The theorem has been proved.

Theorem 10. Let $G$ be a torsion-free hyperbolic group. Then the word and conjugacy problems are solvable in $G^{\mathbf{Q}}$.

Proof. Let $W$ and $V$ be arbitrary $\mathbf{Q}$-words in the alphabet $A$. By Theorem 9 and Lemma 28, one can effectively find a number $n=n(W, V)$ such that the words $W, V$ represent some elements $g, h \in T_{n}$ of $G^{\mathbf{Q}}$. By Theorem 8 the group $T_{n}$ is hyperbolic, and there is an algorithm to construct a finite presentation of $T_{n}$. As was noticed in Lemma 23, if $g, h$ are conjugate in $G^{\mathbf{Q}}$ then they are conjugate in $T_{n}$. So we have reduced the word and conjugacy problems to the hyperbolic case. But in hyperbolic groups the above-mentioned problems are decidable. This completes the proof of the theorem.

\section{REFERENCES}

[1] G Baumslag. On free D-groups, Comm. Pure Appl. Math. 18 (1965), 25-30. MR 31:1293

[2] Some aspects of groups with unique roots, Acta Math. 104 (1960), 217-303. MR 23:A191

[3] G. Baumslag, S. M. Gersten, M. Shapiro, and H. Short, Automatic groups and amalgams, J. Pure Appl. Algebra 76 (1991), 229-316. MR 93a:20048

[4] - Automatic groups and amalgams - a survey, In Algorithms and Classification in Combinatorial Group Theory, Math. Sci. Res. Inst. Publ., vol. 23, Springer-Verlag, Berlin, 1992, pp. 179-194. MR 94g:20040

[5] M. Bestvina and M. Feighn, A combination theorem for negatively curved groups, J. Diff. Geom. 35 (1992), 85-101. MR 93d:53053

[6] S. M. Gersten and H. B. Short, Rational subgroups of biatomatic groups, Ann. of Math. (2) 134 (1991), 125-158. MR 92g:20092

[7] R. Gitik, On combination theorems for negatively curved groups, Internat. J. Algebra Comput. 6 (1996), 751-760. CMP 97:04

[8] M. Gromov, Hyperbolic groups, Essays in Group Theory, Math. Sci. Res. Inst. Publ., vol. 8, Springer-Verlag, Berlin, 1987, pp. 75-263. MR 89e:20070

[9] I. Kapovich, On a theorem of G. Baumslag, Proc. Special Session Combinatorial Group Theory and Related Topics (Brooklyn, NY, 1994), Amer. Math. Soc., Providence, RI (to appear).

[10] W. Magnus, A. Karras, and D. Solitar, Combinatorial group theory, Interscience, New York, 1966. MR 34:7617 
[11] K. V. Mikhajlovskiı̌ and A. Yu. Ol'shanskiŭ, Some constructions relating to hyperbolic groups, 1994, Proc. Int. Conf. on Cohomological and Geometric Methods in Group Theory (to appear).

[12] A. G. Myasnikov and V. N. Remeslennikov, Exponential groups. II: Extension of centralizers and tensor completion of csa-groups, Internat. J. Algebra Comput. 6 (1996), 687-712. CMP 97:04

[13] A. Yu. Ol'shanskǔ, Periodic factor groups of hyperbolic groups, Math. USSR Sb. 72 (1992), 519-541. MR 92d:20050

[14] _ On residualing homomorphisms and G-subgroups of hyperbolic groups, Internat. J. Algebra Comput. 3 (1993), 365-409. MR 94i:20069

[15] P. Papasoglu, Geometric methods in group theory, Ph.D. thesis, Columbia Univ., New York, 1993.

[16] M. D. Shapiro, Automatic structure and graphs of groups, Topology '90, Ohio State Univ. Math. Res. Inst. Publ., vol. 1, de Gruyter, Berlin, 1992, pp. 335-380. MR 93i:20044

Department of Mathematics and Statistics, McGill University, Montréal, P.Q., Canada H3A 2K6

E-mail address: olga@triples.math.mcgill.ca

Department of Mathematics, City College (CUNY), New York, New York 10031-9100

E-mail address: alexei@rio.sci.ccny.cuny.edu 